\title{
Novel Targets in Reducing Cardiac Fibrosis as a Preventive Tool for Cardiovascular Diseases. Why Always Treat Them? A Review Article
}

\author{
Kaleab Gizaw \\ Institute of Health Sciences Jimma University
}

\begin{abstract}
Cardiovascular diseases are the primary causes of death worldwide. These days its mortality and deadliness is rising dramatically. Cardiac fibrosis, one of the leading causes of heart diseases, is a common pathological process in cardiac disease. It can also cause sudden death even in those without cardiac symptoms. Experimental studies in animals have identified important pathways such as the Renin-Angiotensin-Aldosterone System (RAAS) and the endothelin pathway that contribute to fibrosis formation. Currently different groups of drugs are used for treatment of cardiac fibrosis including antihypertensive, anti-inflammatory and cardioprotective agents. Despite the availability of these agents, the prevalence of heart failure and resistant hypertension is increasing. Therefore the search for novel targets is not a questionable issue. Recently different studies are being conducted for treatment of cardiac fibrosis specifically acting on epigenetics (ex. MicroRNAs), Receptors (ex. Adenosine receptors), Enzymes (ex. Lysl Oxidase like 2) and Oxidative stress (ex. N-Acetylcysteine). Therefore the current review looks for promising novel targets aiming at reversing cardiac fibrosis as a preventive tool for cardiovascular diseases.
\end{abstract}

Keywords: Cardiac fibrosis, cardiovascular disease, Novel targets

DOI: $10.7176 /$ JNSR/9-17-03

Publication date:September $30^{\text {th }} 2019$

$\begin{array}{ll}\text { Abbreviations } & \\ \text { ANG } & \text { Angiotensin } \\ \text { CF } & \text { Cardiac Fibrosis } \\ \text { CHF } & \text { Congestive Heart Failure } \\ \text { ECM } & \text { Extra Cellular Matrix } \\ \text { ET-1 } & \text { Endothelin type 1 } \\ \text { IL } & \text { Interleukin } \\ \text { LOXL2 } & \text { Lysyl Oxidase-Like 2 } \\ \text { MI } & \text { Myocardial Infarction } \\ \text { MiRNA } & \text { Micro Ribo Nucleic Acid } \\ \text { NAC } & \text { N-Acetylcysteine } \\ \text { RAAS } & \text { Renin Angiotensin Aldosterone System } \\ \text { ROS } & \text { Reactive Oxygen Species } \\ \text { TGF- } \beta & \text { Transforming Growth Factor Beta } \\ \text { TNF- } \alpha & \text { Tissue Necrosis Factor alpha }\end{array}$

\section{Background}

\subsection{Introduction}

Cardiovascular diseases (CVDs) are the primary causes of death worldwide, with 17.5 million deaths from CVD in 2012 representing 31\% of all global deaths that year (Duygu et al., 2013). Fibrosis appears as a common pathophysiological phenomenon, especially during the development and progression of cardiovascular diseases. The normal cardiac anatomy is progressively modified by the excessive deposition of extracellular matrix with reduction of microvasculature and disruption of normal myocardial structures (Porter and Turner, 2009, Roubille et al., 2014, and M.Gyongyosi et al., 2017).

Myocardial / Cardiac fibrosis refers to a variety of quantitative and qualitative changes in the interstitial myocardial collagen network that occur in response to cardiac ischemic insults, systemic diseases, drugs, or any other harmful stimulus affecting the circulatory system or the heart itself (M.Gyongyosi et al.,2017).

It is a result of a variety of injurious insults of different causes to cardiac tissue, which ultimately culminates in destruction of physiological tissue architecture and progressive organ dysfunction. Histologically, it is characterized by activation/proliferation of fibroblasts and excessive matrix deposition, including collagen (Roubille et al., 2014 and M.Gyongyosi et al., 2017).

Cardiac fibrosis leads to irreversible death of myocardial cells and is associated with increased stiffness that contributes significantly to diastolic dysfunction (Porter and Turner, 2009, Biernacka et al.,2011 and Roubille et al., 2014), whereas regression of fibrosis is believed to improve cardiac function (Jalil et al.,1989 and Roubille et 
al., 2014).

Myocardial fibrosis is a significant global health problem associated with nearly all forms of heart diseases including heart failure (Biernacka et al., 2011, Roubille et al., 2014, Travers et al., 2016 and Adebiyi et al.,2016), myocardial infarction (Jalil et al.,1989) and cardiac arrhythmias (Hrayr et al.,2011, Travers et al., 2016 and M.Gyongyosi et al.,2017) among others.

Myocardial fibrosis (MF) is a distinct structural feature of cardiac remodeling and a common finding in the histopathological examinations of the myocardium and a predisposing risk factor for the development of heart failure in patients in type 1 diabetes mellitus as well (Adebiyi et al.,2016 and Norshige et al.,2011).

Increased fibrosis has been observed around trabeculae and in the interstitial spaces of the atrial myocardium during Congestive heart failure (CHF) in both animal models and human biopsies. Moreover, there are profound changes in the atrial electrical properties including depressed excitability, increased refractoriness, and conduction slowing or block, which have been related to the degree of interstitial fibrosis. This atrial remodeling is likely responsible for the composition of the substrate for atrial fibrillation (AF), the incidence of which is increased during CHF (M.Gyongyosi et al., 2017 and Hrayr et al., 2011).

\subsection{Classification of cardiac fibrosis}

Cardiac fibrosis can be categorized into two types: reactive fibrosis and replacement fibrosis (also called reparative fibrosis). The term "reactive interstitial fibrosis" is used to describe expansion of the cardiac interstitial space in the absence of significant cardiomyocyte loss; in contrast "reparative fibrosis" refers to the formation of a scar in response to myocardial infarction. Replacement fibrosis often occurs after Myocardial Infarction (MI) when large numbers of cardiac myocytes undergo necrosis. Myocyte necrosis triggers a series of events including immune cell infiltration, inflammation, new vessel formation, removal of necrotic tissue, and eventually the replacement of damaged tissue with collagen dominated fibrotic tissue that prevents cardiac muscle from rupture. In addition to scar formation at the infarcted area, the remote noninfarcted regions can develop fibrosis in the interstitial spaces, which is referred to as reactive fibrosis or interstitial fibrosis. Interstitial fibrosis can also occur in disease conditions that often involve the activation of Renin-Angiotensin-Aldosterone System (RAAS), TGF- $\beta$, TNF- $\alpha$ or other profibrotic signaling pathways (Weber et al., 1992 and Prabu and Frangogiannis, 2016).

It can either be localized which is mostly evidenced by scarring following myocardial infarction [MI]), or diffuse which is commonly seen in dilated cardiomyopathy) (Jalil et al., 1989 and Biernacka et al., 2011).

\subsection{Pathogenesis}

Tissue fibrosis is characterized by an accumulation of proteins, especially collagen, in the extracellular space, which are secreted by profibrotic cells such as fibroblasts. As a result of the excessive accumulation of proteins, nearby cells become hypotrophic and less numerous and their normal activity is hampered (Roubille et al., 2014 and Anderson et al., 1979).

Cardiomyocytes, fibroblasts, and vascular cells in the heart are connected by a complex matrix principally composed of fibrillar collagen, which is instrumental in preserving structural integrity and plasticity. In the diseased heart, the matrix undergoes structural and sub cellular changes that progressively influence heart function. Beyond the cardiomyocyte-centric view of heart injury, it is now accepted that alterations of the cardiac extracellular matrix (ECM) and cardiac remodeling play a major role in the development and evolution of cardiac diseases leading to heart failure (HF) and other cardiovascular diseases (Travers et al.,2016 and Biernacka et al.,2011).

Myocardial fibrosis is responsible for alteration of level of soluble collagen. There are three different types of collagen that shows accumulation due the generation of myocardial fibrosis. Type I collagen showed a high level as compared to the others. Type I collagen is responsible for stiffening of ventricles which further alters myocardial autorhythmic activity and contraction (Thomas et al., 2007).

Cardiac fibroblasts are derived from epithelial and endothelial cells during embryonic development of the heart in a process called epithelial mesenchymal transition (EMT) and endothelial-mesenchymal transition (EndMT) respectively (Turner et al., 2009, Prabu and Frangogiannis, 2016 and Thomas et al., 2007).

Resident cardiac fibroblasts are considered the major source but other cell types such as vascular endothelial cells, perivascular cells, and progenitor cells have been shown to differentiate into myofibroblasts (Travers et al., 2016 and Thomas et al., 2007).

Fibrosis develops when the body's natural wound-healing process goes awry. Under normal (adaptive) conditions of wound healing, specialized cells known as fibroblasts become activated by transforming to myofibroblast. The myofibroblasts then undergo proliferation causing increased synthesis of collagen protein in the extracellular matrix composed predominantly of type I collagen and to a lesser extent type III collagen (Thomas et al., 2007).

In addition, myocardial fibrosis is characterized by dysregulated collagen turnover (increased synthesis predominates over unchanged or decreased degradation) and excessive diffuse collagen accumulation in the 
interstitial and perivascular spaces. This dysregulation of collagen turnover takes place mainly in phenotypically transformed fibroblasts, termed myofibroblasts. (Norshige et al., 2011)

Cardiac fibroblasts differentiation into myofibroblasts

Cardiac fibroblasts have greater quantities than cardiomyocytes in the heart tissue. They are quiescent in healthy heart tissue, and are responsible for ECM secretion to keep the integrity of the interstitial matrix. After MI, the death of cardiomyocytes activates immune response that induces cytokine and chemokine expression. This initiates the infiltration of neutrophils and mononuclear cells to the infarcted area. The neutrophils are then phagocytosed by macrophages after apoptosis. The macrophages are able to secrete profibrotic cytokines like TGF- $\beta$, Angiotensin II, and platelet-derived growth factor (PDGF). TGF- $\beta$ binds to TGF- $\beta$ receptors type I and II, and activates the TGF- $\beta /$ Smad pathway to differentiate the cardiac fibroblasts into myofibroblasts (Shinde et al., 2014). TGF- $\beta$ has been demonstrated as a major mediator of myofibroblast formation after MI. The formed myofibroblasts then produce excessive ECM to initiate the cardiac fibrosis (Shinde et al., 2014 and Leask et al., 2015).

Following myofibroblast differentiation, its number increases over a period of a few months in the infarcted area. More ECM is thus generated and deposited, leading to the increase of scar size. In the scar, the content of collagen type III-rich fibers increases in a few weeks. The fibers are then gradually replaced by stiffer type I collagen. The scar tissue matures when the collagen fibers are crosslinked. Unlike other scar tissues, myofibroblasts exist in the cardiac scar for many years, and continue generating ECM (Leask et al., 2015).

Fibrocytes differentiation into myofibroblasts

Fibrocytes are a type of fibroblast-like peripheral cells. These cells express fibroblast specific proteins, cluster of differentiation 31 and 45 (CD34 and CD45). In response to chemokines such as chemokine ligand 21 and chemokine ligand 12, fibrocytes migrate towards the injured area. Under the stimulation of TGF- $\beta$ or endothelin1 , fibrocytes differentiate into myofibroblast type cells with expression of $\alpha$-smooth muscle actin, production of fibronectin and collagen, and loss of expression of CD34 and CD45. Besides TGF- $\beta$ and endothelin-1, cytokines including Interleukin-13, Interleukin-14 and Platelet Derived Growth Factor (PDGF) also promote the fibrocytes to differentiate into myofibroblasts (Mori et al., 2005).

Epithelial to mesenchymaltransdifferentiation

Epithelial to mesenchymaltransdifferentiation (EMT) is another origin of myofibroblasts, which is a process of transdifferentiation from epithelial cells into myofibroblast-like cells. In the EMT process, the expression of mesenchymal marker is up-regulated while the expression of epithelial marker is downregulated. TGF- $\beta 1$ plays a key role in this process. While the roles of other cytokines in EMT are still in debate, strong evidences suggest that TNF $\alpha$ and Interleukin- $1 \beta$ are capable of accentuating the effect of TGF- $\beta 1$ in driving EMT (Yamauchi et al., 2010).

Endothelial to mesenchymal transition

While resident cardiac fibroblasts may be activated and transformed to myofibroblasts there is also the potential of fibroblasts originating from endothelial cells, suggesting an endothelial-mesenchymal transition (EndMT). For example, it has been shown that TGF- $\beta 1$ induces endothelial cells to undergo EndMT, whereas bone morphogenic protein 7 (BMP-7) preserves the endothelial phenotype (Zeisberg et al., 2007).

Endothelial to mesenchymal transition (EnMT) is currently thought to be a potential origin of myofibroblasts. EnMT was first proposed to be a phenomenon related to embryonic development until evidence indicated that EMT, EnMT can be driven by TGF- $\beta$ (Types 1 and 2) and be augmented by TNF- $\alpha$ and IL-1 $\beta$ (Maleszewska et al., 2013).

Cardiac fibroblast proliferation and differentiation into myofibroblasts are important processes in both types of cardiac fibrosis (Baudino et al., 2006). Myofibroblasts, which are called the activated cardiac fibroblasts, are considered as the major source that secret collagen and other extracellular matrix (ECM) proteins during the formation of fibrosis (Travers et al., 2016, Baudino et al., 2006, and Susane et al., 2010).

\subsection{Signaling pathways and their association with Cardiac Fibrosis}

The RAAS and TGF- $\beta$ signaling pathways are major factors that are involved in the activation of cardiac fibroblasts and formation of tissue fibrosis. Administration of angiotensin II or aldosterone has been shown to stimulate collagen and other ECM protein expression and secretion from cardiac fibroblasts (Lijnen et al., 2000). Several members of the TGF- $\beta$ family are involved in the regulation of inflammation and fibrosis (Euler et al., 2015). The TGF- $\beta 1$ signaling pathway is an important component in the switch from inflammation to fibrosis (Dobaczewski et al., 2011).

In chronic conditions, activation of RAAS, TGF- $\beta$ and other pro-fibrotic signaling pathways can induce interstitial fibrosis in the heart and other organs (Piera-Velazques et al., 2011).

In the event of an MI, activation of NFKB was observed in different cells and subsequently drives the expression of a large panel of genes. These genes produce pro-inflammatory cytokines such as Tumor Necrosis Factor- $\alpha(\mathrm{TNF}-\alpha)$ and interleukin proteins that subsequently help recruit leukocytes and initiate the inflammation 
responses (Prabu and Frangogiannis, 2016 and Sun et al., 2007).

In addition to these traditional signaling pathways, epigenetic regulation has drawn attention for their regulating role in cardiac fibrosis. Numerous MicroRNAs were found to be either pro- or anti-fibrotic (Lakatta et al., 2003). Some anti-fibrotic MicroRNAs such as the miR-29 microRNA family directly target the mRNA of several collagen isoforms, fibrilin 1, elastin, and matrix metalloprotease 2 (MMP2) and thus prevent over expression of these ECM proteins (Stein et al., 2010). When the anti-fibrotic MicroRNAs are reduced in disease state, syntheses of collagen and other ECM protein increase and promoting the development of fibrosis.

The role of the Renin-Angiotensin-Aldosterone System (RAAS)

A large body of evidence indicates that the activation of Renin-Angiotensin-Aldosterone System (RAAS) might play a central role in cardiac aging and in age-associated fibrotic remodeling. Even in the absence of overt hypertension, arterial vascular walls become less compliant with age, resulting in some degree of pressure overload. ANG II concentrations increase significantly in aged the heart, probably due to an increase tissue level of angiotensin II converting enzyme (ACE). ANG II promotes cardiomyocyte hypertrophy (Euler et al., 2015 and Ventura Clapier et al., 2008) and stimulates fibroblast proliferation and expression of extracellular matrix proteins.

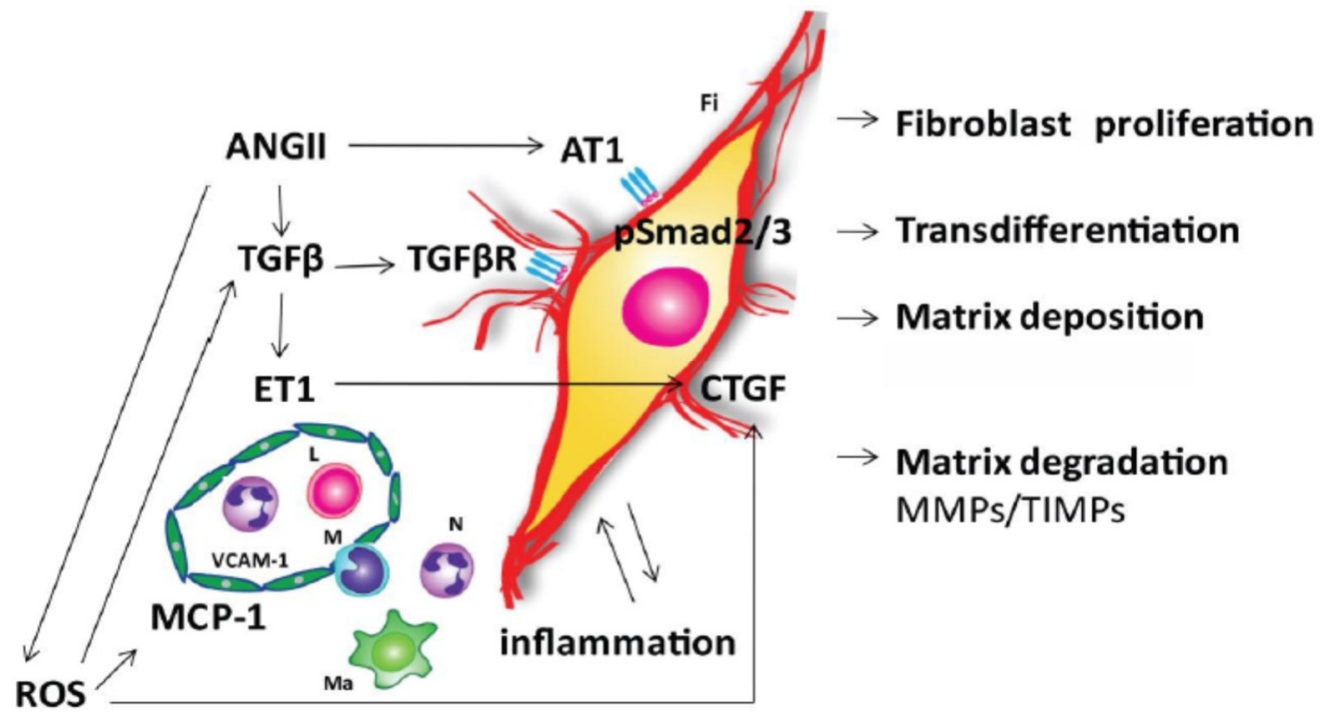

Figure 1. Pathways involved in the pathogenesis of cardiac fibrosis in the senescent heart, the role of Angiotensin II Reactive Oxygen Species (ROS) (Adopted from Biernacka et al., 2011)

The role of reactive oxygen species (ROS)

Within the cells, ROS are produced in multiple compartments; however, mitochondria contribute to the majority of ROS generation as a byproduct of electron transfer during oxidative phosphorylation. Mitochondrial DNA (mtDNA), lipids, and proteins are therefore at the highest risk from free radical-induced damage and dysfunction. Several studies have documented an age related impairment of mitochondrial function associated with increased production of ROS. The heart, with its high metabolic demand is rich in mitochondria and is especially vulnerable to mitochondrial oxidative damage. Impairment of mitochondrial function has been widely documented in heart failure in both human patients and mouse models (Ventura Clapier et al., 2008). Moreover a significant increase in superoxide radical production was seen in mitochondria prepared from aging rat hearts.

The role of TGF- $\beta$

TGF- $\beta$ may play an essential role in cardiac fibrosis by inducing myofibroblasttransdifferentiation and by enhancing matrix protein synthesis by cardiac fibroblasts (Bujak et al., 2007). In addition, TGF- $\beta$ may exert potent matrix-preserving actions by suppressing the activity of mixed metalloproteases and by inducing synthesis of protease inhibitors, such as PAI-1 and TIMPs. Beyond its effects on mesenchymal cells, TGF- $\beta$ also induces hypertrophic effects on cardiomyocytes and modulates the function of inflammatory cells (Shi et al., 2003).

Both ROS and ANG II may activate TGF- $\beta$ signaling pathways in the senescent heart. ROS activate TGF- $\beta$ and upregulate its downstream fibrogenic effector, connective tissue growth factor (CTGF). In addition, ANG II markedly upregulates TGF- $\beta 1$ synthesis by cardiac fibroblasts and myofibroblasts. ANG II-induced TGF- $\beta$ upregulation is followed by the development of cardiac fibrosis (Rosenkranz et al., 2004).

The role of $\beta$-adrenergic signaling

Activation of $\beta$-adrenergic signaling increases heart rate, contractility and afterload, enhancing cardiac metabolic demand. Chronic activation of $\beta$-adrenergic signaling is deleterious to the heart; several clinical trials have demonstrated that inhibition of $\beta$-adrenergic signaling by $\beta$-blockers provides survival benefit in patients with heart failure. Mice with disruption of adenylatecyclase type 5 (AC5), a major mediator of $\beta$-adrenergic signaling 
in the heart, had prolonged life span and were protected from cardiac aging, exhibiting reduced age-dependent cardiac hypertrophy and attenuated fibrosis (Stein et al.,2010 and Rosenkranz et al.,2002).

TGF- $\beta 1$-overexpressing mice exhibited enhanced $\beta$-adrenergic signaling and significant cardiac hypertrophy accompanied by interstitial fibrosis. On the other hand, loss of one TGF- $\beta 1$ allele in TGF- $\beta 1$ heterozygous mice appears to ameliorate age-associated myocardial fibrosis and improve left ventricular compliance (Brooks et al., 2000).

The role of Endothelin

The endothelin family of peptides is typically recognized for its vasoconstrictive properties and is now beginning to be appreciated for its potential role in promoting tissue fibrosis. Although originally thought to be secreted predominantly by endothelial cells, endothelin-1 (ET-1) is now understood as a significant profibrotic peptide released by both inflammatory cells and fibroblasts in the lung (Shi-Wen et al., 2006).

There exist two known receptors for ET-1 in the heart, the ETA and ETB receptors, which have been shown to play differing and sometimes opposing roles. These receptors are primarily expressed by endothelial cells; however, new data define expression on multiple cell types including cardiomyocytes and cardiac fibroblasts, along with some immune cells, such as macrophages (Chen et al., 2006).

Importantly, ET-1 activation of the ETA receptor is known to increase collagen production in isolated human cardiac fibroblasts. Furthermore, myofibroblasts isolated from scar tissue after experimental MI possess elevated levels of ET-1, suggesting an important function for endothelin within these cells. Although known to have fibroblast-activating properties of its own, ET-1 also acts as a downstream mediator of both ANG II and TGF $\beta$ to promote myofibroblast persistence in pulmonary fibrosis (Dashwood et al., 2011).

ET-1 signaling is thought to interact with ANG II as well. For example, the development of cardiac fibrosis and hypertrophy in response to ANG II stimulation is impaired in mice in which ET-1 is ablated in endothelial cells (Hafizi et al., 2004).

Endothelin antagonists are currently approved for the treatment of pulmonary hypertension, and many think they will be additionally efficacious in the treatment of pathological fibrosis in the heart. Bosentan, a nonselective endothelin receptor antagonist, although used clinically for the treatment of pulmonary hypertension, can improve cardiac function and reduce infarct size after myocardial ischemia/reperfusion injury in rats. Next generation endothelin receptor subtype-specific antagonists, such as Ambrisentan and Darustentan that are proving to be highly beneficial in the treatment of pulmonary hypertension, may prove more efficacious and help define receptor specificity that may be required for salutary effects (Katwa et al.,2003).

\section{Currently available treatment approaches targeting cardiac fibrosis \\ 2.1 Antihypertensive agents}

In vivo and in vitro experiments have shown that the effector hormones of the Renin angiotensin- aldosterone system, angiotensin II and aldosterone stimulate fibroblast-mediated collagen synthesis (Brilla et al., 1994).

Most of the tested antihypertensive therapies have been shown to reduce fibrosis in animal models. In a rat model of aortic banding associated with renal artery ligation, an Angiotensin converting enzyme (ACE) inhibitors, a $\beta$-blockers and a calcium-channel blocker all reduced left ventricular (LV) fibrosis (Brilla et al., 2000).

However, anti-hypertensive drugs do not all seem to exert similar effects in patients. In hypertensive patients treated with either Lisinopril $(n=18)$ or hydrochlorothiazide $(\mathrm{HCTZ})(\mathrm{n}=17)$, endomyocardial biopsies at baseline and 6 months revealed a decrease of collagen volume fraction (CVF) only in the group treated with the ACE inhibitor. With Lisinopril, CVF was reduced significantly, whereas no regression of myocardial fibrosis occurred with Hydrochlorothiazide (Funck et al., 2000).

A comparison between Losartan $(n=21)$ and Amlodipine $(n=16)$ given for one year revealed that while changes in blood pressure during treatment were similar in the two groups, only Losartan resulted in significant decrease in CVF (Lopez et al.,2001).

\subsubsection{Angiotensin Converting Enzyme Inhibitors (ACEi)}

Angiotensin II suppresses the collagenase activity that synergistically leads to progressive collagen accumulation within the cardiac interstitium (Brilla et al., 1994, Brilla et al., 2000, Funck et al., 2000 and Lopez et al., 2001). Angiotensin-converting enzyme inhibitors remain a first-line treatment for hypertension. The ACE inhibitor Lisinopril has been shown to induce regression of myocardial fibrosis in rats with genetic hypertension and LV hypertrophy (Brilla et al., 1996).

In spontaneously hypertensive rats, LV hypertrophy and myocardial fibrosis could be regressed and LV diastolic function could be improved by treatment with the angiotensin-converting enzyme (ACE) inhibitor Lisinopril (Funck et al., 2000 and Brilla et al., 1996).

ACE inhibitors can prevent the LV remodeling process that accompanies cardiac dysfunction after MI. These findings suggest that ACE inhibitors prevent LV remodeling after MI by mechanisms other than inhibition of angiotensin AT1 receptor mediated effects (Yoshiyama et al., 2005). 


\subsubsection{Angiotensin Receptor Blockers (ARBs)}

ARBs have also been shown to have favorable effects on ventricular fibrosis. Losartan and Olmesartan have improved healing after MI in rodents ((Scheiffer et al., 1994, Matsusaka et al., 2006). ARBs have also been shown in small clinical studies to reduce fibrosis biomarkers. For example, Candesartan reduced fibrosis biomarkers in 153 patients with atrial fibrillation (this could reflect either atrial or myocardial fibrosis reduction) (Kawamura et al., 2010).

Irbesartan, another ARB possesses anti-fibrotic activity and offers protection against myocardial fibrosis, due to regulation of angiotensin II activity through blockade of Angiotensin (AT1) receptors which might attenuate the endoplasmic reticulum stress and thus inhibit myocardial fibrosis via inhibition of oxidative stress. The regulation of antioxidant defensive enzymes may be involved in anti-fibrotic effect of Irbesartan (Vandaria et al., 2015).

\subsubsection{Aldosterone antagonists}

The mineralocorticoid receptor antagonist Spironolactone has been shown to be a promising drug in animal models and has been recently proposed in clinical conditions associated with fibrosis like metabolic syndrome and obesity.

A comparison between Spironolactone $25 \mathrm{mg} /$ day and placebo for 6 months in 80 patients with metabolic syndrome already treated with ANG II inhibition revealed that only the Spironolactone treated group showed significant improvement of LV diastolic function and parallel decreases in PICP and PIIINP levels (Azibani et al.,2012 and Kosmala et al.,2011).

Interesting results have also been obtained in a small study of 44 patients with diastolic HF who were treated with either placebo or the mineralo-corticoid receptor antagonist Eplerenone (Mak et al., 2009).

A study comparing the percentage of fibrosis between groups taking Spironolactone, Lisinopril and Atenolol using left ventricular end-diastolic pressure (LVEDP) as the covariate, Spironolactone proved to be the most effective drug for the reversal of fibrosis (Paul et al.,2005).

A study conducted regarding the effect of Potassium Canrenoate, an aldosterone antagonist in reducing Isoprenaline induced fibrosis revealed that antagonism of aldosterone receptors with Potassium Canrenoate interacts with cardiac remodeling after the acute administration of high doses of Isoprenaline. The drug reduces the extent of fibrosis and such effect is associated with a greater left ventricular enlargement (Bos et al., 2004).

\subsection{Anti-inflammatory agents}

Targeting some of the most powerful pro-inflammatory cytokines (TNF- $\alpha$, IL-1 and IL-6) could have beneficial effects on cardiac fibrosis. The TNF- $\alpha$ pathway is clearly involved in cardiac fibrosis in various basic models (Duerrschmid et al., 2013).

\subsubsection{Galectin 3 Inhibitors}

Galectin-3 is a member of the lectin family and has been shown to be involved in inflammation, cell adhesion and activation, including in heart diseases. It was found to be over expressed in failing hearts in a rat model of HF. Myocardial biopsies revealed that galectin-3 was over expressed at an early stage specifically in the rats that later developed HF. Galectin-3 was proposed to represent a biomarker for activated macrophages, but could also be involved in activation of cardiac fibroblasts. Importantly, recombinant galectin-3 induced cardiac fibroblast proliferation, collagen production and an increase of collagen I over collagen III (De Boer et al., 2010 and Sharma et al., 2004). Sustained delivery of drugs that decrease inflammation in the infarcted hearts after MI may decrease the inflammation cytokines-associated myofibroblast formation, thereby reducing cardiac fibrosis (Sharma et al., 2004).

\subsubsection{Ibuprofen}

Ibuprofen is a cyclo-oxygenase inhibitor with anti-inflammatory property. It directly inhibits leukocyte activation and accumulations, and decreases the production of leukocyte attractant and activator leukotriene B4. Vu et al. injected ibuprofen-containing hyaluronic acid hydrogel into infarcted pig hearts, and found that cardiac fibrosis was significantly decreased compared to the hydrogel only group (Vu et al., 2015).

\subsection{Selective heart rate-reducing agents}

\subsubsection{Ivabradine}

$\beta$ - blockers are groups of drugs that inhibit cardiac fibroblast growth and hence inhibit cardiac fibrosis (Turner et al., 2003). The If current inhibitor Ivabradine provides selective heart rate reduction and has recently been introduced in the treatment guidelines for HF in patients with LV systolic dysfunction (Roubille et al.,2013).

In a rabbit model of diastolic dysfunction, Ivabradine attenuated LV diastolic dysfunction and also reduced significantly both atrial and ventricular fibrosis as well as ventricular collagen type I. Interestingly, Ivabradine also decreased plasma ANG II levels in that study (Busseuil et al.,2010).

In support of this notion, Ivabradine was found more efficacious to reduce cardiac fibrosis than Metoprolol in mice models of HF (Becher et al., 2012). Ivabradine could reduce systemic inflammation and pro-inflammatory cytokines (Li et al., 2013). 
Both Ivabradine and Carvedilol similarly attenuated myocardial lesions and fibrosis, inhibited nitric oxide (NO) synthesis by inducible NOS, and decreased the production of TNF- $\alpha$ and IL- 6 . Beta-blockers could also be of interest, through the reduction of HR and blood pressure, but also by other effects including NO production and anti-oxidative action as has been reported for the beta-blocker Carvedilol (Leki et al., 1989 and Bartholomeu et al., 2008).

\section{Novel treatment targets of cardiac fibrosis 3.1 Epigenetic based therapies 3.1.1 MicroRNAs}

MicroRNAs (miRNAs) are short, noncoding RNAs ( $\approx 22$ nucleotides) that are formed in a highly regulated process in the nucleus and are then transported into the cytosol, in which they are processed further (Lorenzen et al., 2012 and Quiat et al., 2013).

MiRNAs modify gene expression by down regulating genes at the post-transcriptional level during various developmental or disease processes. These $\approx 22$ nucleotide RNAs bind to their targets in the 3' untranslated region (UTR) of messenger RNAs (mRNAs) to inhibit translation or evoke degradation of the mRNA (Quiat et al., 2013 and Napoli et al., 2016). MiRNAs are highly conserved in different species and are thought to regulate $50 \%$ of the genome. In cardiac development, miRNAs are needed for the formation of normal, functional heart tissue (Napoli et al., 2016)

Initially, primary miRNAs (pri-miRNA) are generated in the cellular nucleus by the transcription machinery and are then processed by the RNase-III-type enzyme Drosha to form so called precursor miRNAs (pre-miRNAs). Following exportation into the cytoplasm, miRNAs are processed by the ribonuclease Dicer into small 20-23 nucleotide long miRNA duplexes. Finally, miRNAs are incorporated into RNA Induced Silencing Complexes (RISC) to silence gene expression at the post-transcriptional level by targeting messenger RNAs (mRNAs) with the result of mRNA degradation or by translational inhibition finally leading to target protein repression (Lorenzen et al., 2012,Quiat et al., 2013, Napoli et al., 2016 and Ooi et al.,2016).

With miRNAs highly integrated into every cellular event, the potential impact on cardiac function is profound when considering the ramifications of misregulation of processes such as fibrosis. Upregulation of miRNAs may be beneficial for normalizing disease states; however, over expression may tilt the balance in the wrong direction (Quiat et al., 2013, Bauersachs et al., 2016 and Liang et al., 2012).

MicroRNAs have recently come into focus as powerful regulators of gene expression, and they fundamentally influence the pathogenesis of different pathological events, including cardiac fibrosis. The miRNA expression patterns change in various cardiovascular diseases, such as myocardial infarction, cardiac hypertrophy and heart failure (Bauersachs et al., 2016).

Non-myocyte myocardial cells, such as endothelial cells and fibroblasts, play a key role in remodeling processes, contributing to the development of heart failure. Among others, miR-21 and miR-29 have been studied most intensively in relation to cardiac fibrosis (Liang et al., 2012).

Cardiac fibroblasts are enriched with certain miRNAs, such as miR-21 (Liu et al., 2010 and Zhong et al., 2011), which regulates the ERK-MAP kinase signaling pathway via targeting sprouty-1. By this mechanism, fibroblast survival and growth factor secretion, apparently regulating the extent of interstitial fibrosis and cardiac hypertrophy, is controlled. In vivo silencing of miR-21 by a specific cholesterol-based antagomir in a mouse pressure-overload-induced disease model reduced cardiac ERK-MAP (Extracellular signal Regulated Kinase Mitogen Activated Protein) kinase activity, inhibited interstitial fibrosis and attenuated cardiac dysfunction. In addition, recently it has been shown that miR-21 antagonism blocks endothelial-mesenchymal transition in TGF$\beta$ treated endothelial cells as well as in vivo in a cardiac hypertrophy model resulting in reduced fibrosis development. Strikingly, the effects of miR-21 inhibition on fibrosis prevention were validated in other organs often affected by pathological fibrosis during diseases such as the lung (Liu et al., 2010) and the kidney (Volkmann et al., 2012). Thus, miR-21 inhibition may represent a general approach to inhibit exaggerated organ fibrosis.

MiR-21 was also associated with the TGF- $\beta$ pathway and TGF- $\beta$ receptor, in which over expression of miR21 led to increased cardiac fibrosis due to the TGF- $\beta$ mediated endothelial-to-mesenchymal transition. This miRNA was upregulated in the mouse myocardium after cardiac stress and in the myocardial tissue of patients with aortic stenosis. In these patients, miR-21 was only present in interstitial cells and correlated with collagen expression in the heart (Zhong et al., 2011 and Volkmann et al., 2012).

In addition, in cardiac fibrosis in response to cardiac hypertrophy and failure, miR-21 is specifically enriched in cardiac fibroblasts and regulates the ERK - MAP kinase signaling pathway through inhibition of sprout homolog 1. By this mechanism, miR-21 promotes fibroblast survival and growth factor secretion. In contrast, silencing of miR-21 with a specific antagonist (antagomir) culminated in a reduction of cardiac extracellular signal-regulated kinase- mitogen-activated protein kinase activity, inhibition of interstitial fibrosis, and improvement of cardiac dysfunction (Liang et al., 2012, Zhong et al., 2011 and Volkmann et al., 2012).

Wang et al. showed that miR-24 could interfere with TGF signaling by targeting the pro-protein convertase, 
furin, and subsequently down regulate the level of TGF in cardiac fibroblasts (Wang et al., 2012).

MiR-29 was identified to target several collagens, including collagen type 1A1, collagen type 1A2, and collagen type 3A1, as well as fibrillin 1, thus promoting extracellular matrix deposition after myocardial infarction (MI). Specifically, after MI, the miR-29 family is downregulated in vivo, thereby derepressing its targets and resulting in cardiac fibrosis (Van Rooij et al., 2008).

In a pioneer study by Olson's group, expression of miR-29 family members (miR-29a, miR-29b and miR29c) negatively correlated with expression of genes involved in ECM production and fibrosis after experimental myocardial infarction. Furthermore, the miR-29 family regulates the expression of pro-fibrotic genes, including extracellular matrix genes elastin, fibrillin 1 (Fbn1), collagen type I, 1 and 2 (Col1-1, Col1-2) and collagen type III, -1 (Col3-1) (El Vegteret et al., 2016).

In contrast to a down regulation, during aging, an upregulation of miR-29 was seen in the aorta of mice and the increased expression of miR-29 family members was associated with a profound down regulation of numerous ECM components in aortas of aged mice and during aneurysm formation (Boon et al., 2011). Of clinical relevance, miR-29b levels were also profoundly increased in human thoracic aneurysms.

In vitro knockdown of miR-29 resulted in increased cardiac fibrosis whereas over expression resulted in reduced cardiac fibrosis. In vivo, miR-29b over expression by miR-29b transfection into the heart attenuated the progression of fibrosis and led to an improvement of cardiac function. The TGF- $\beta /$ Smad 3 signaling pathway has been described as playing a crucial role in cardiac fibrosis as target of miR-29 (Zhang et al., 2014 and Zhou et al., 2012).

Thioredoxin, which regulates in part cardiomyocyte hypertrophy, leads to an increase in expression of the let7 family including miR-98. To evaluate the function of miR-98 in the heart in vivo, adenoviral miR-98 or antimiR98 was injected into mouse hearts. ANG II-induced increases in hypertrophy, cardiomyoctye apoptosis and fibrosis were attenuated by Ad-miR-98 and significantly enhanced by AdantimiR-98. MiR-98 negatively regulates ANG II-induced cardiac hypertrophy and the accompanying histopathologic changes in vivo and thus is an attractive target for the treatment of cardiac remodeling. (Yang et al., 2011) MicroRNAs have been demonstrated to be powerful antifibrotic agents (Thum and Lorenzen, 2016).

One of the first studies that used an antagomir to inhibit a MiRNA involved in cardiac hypertrophy was performed by Care and coworkers. This group implanted mice subcutaneously with osmotic minipumps for a continuous delivery of a cholesterol-based antagomir targeting miR-133. After 1 month of infusion, echocardiographic analysis showed a marked increase in cardiac hypertrophy suggesting that miR-133 mimics may be of therapeutic relevance (Thum and Lorenzen, 2016 and Zhao et al., 2015).

In a rat model of MI, the expression of miR-101a and miR-101b (miR-101a/b) in the peri-infarct area was decreased, whereas the cardiac performance improved after adenovirus-mediated over expression of miR-101a as evidenced by echocardiography and hemodynamic measurements (Pan et al.,2012). Importantly, interstitial fibrosis was decreased by the treatment in that study.

Recently, miR-101and miR-101a were found to negatively regulate TGF signaling through targeting of TGF receptor type 1 (TGF-R1) and c-Fos (Nagpal et al., 2016). Cardiac fibrosis is significantly suppressed by upregulation of miR-101a in the rat myocardial infarction model.

Several other miRNAs have been identified as also targeting collagens and TGF_signaling to play a role in fibrogenesis. The role of miR-133a in myocardial fibrosis and electrical depolarization in pressure-overloaded adult hearts may reflect its regulatory effects on the expression of Col1_1, Serca2a and calcineurin (Duisters et al., 2009). MiR-125b is a pro-fibrotic MiRNA via targeting of apelin, one of the key fibrogenesis repressors in the heart (Da Costa et al., 2010).

Another group showed involvement of miRNAs in cardiac hypertrophy; miR-199b is a direct calcineurin/nuclear factor of activated T cells (NFAT) target that increases in expression during mouse and human heart failure thus being a potential interesting target for therapies. Indeed, in vivo inhibition of miR-199b by a specific intraperitoneally administered antagomir normalized reduced nuclear NFAT activity and caused inhibition and even reversal of cardiac hypertrophy and fibrosis in mouse models of heart failure (Da Costa et al., 2010).

In another mouse model, genetic deletion of miR-214 caused loss of cardiac contractility, increased apoptosis and excessive fibrosis in response to ischemia-reperfusion injury (Arin et al., 2012). 
Table 1 Recent evidence investigating the role of circulating miRNAs as biomarkers in several cardiovascular diseases. Taken from (Napoli et al., 2016)

\begin{tabular}{|c|c|c|c|}
\hline miRNAs & Sources & Conditions & Ref. \\
\hline $\begin{array}{l}\uparrow \mathrm{miR}-339-5 \mathrm{p}, \mathrm{miR}-483-3 \mathrm{p} \\
\downarrow \mathrm{miR}-139-5 \mathrm{~b}\end{array}$ & Plasma & LVI & Saddic et al ${ }^{[64]}(2015)$ \\
\hline$\downarrow \mathrm{miR}-145$ & Plasma & AMI & Gao et al ${ }^{[65]}(2015)$ \\
\hline$\uparrow \mathrm{miR}-122, \mathrm{miR}-140-3 \mathrm{p}, \mathrm{miR}-720, \mathrm{miR}-2861, \mathrm{miR}-3149$ & Plasma & ACS, AMI & Li et al $l^{[60]}(2015)$ \\
\hline$\uparrow$ Let-7e, miR-15a, miR-196b & Plasma & AAA, Atherosclerosis & Stather et al ${ }^{[07]}(2015)$ \\
\hline \multicolumn{4}{|l|}{$\operatorname{lmiR}-411$} \\
\hline$\downarrow \mathrm{miR}-125 \mathrm{~b}, \mathrm{miR}-320 \mathrm{~b}$ & Plasma & AMI, CAD & Huang et al ${ }^{[60]}(2014)$ \\
\hline $\mid \mathrm{miR}-21$ & Serum & $\mathrm{CAD}$ & Fan ct al $^{[0]}(2014)$ \\
\hline $\mid \mathrm{miR}-31$ & Plasma & CAD & Wang et al $^{[70]}(2014)$ \\
\hline †miR-146a, miR-186, miR-208b, miR-499 & Serum & ACS, Stable CAD, CV risk & Wu et al ${ }^{[71]}(2014)$ \\
\hline$\uparrow \mathrm{miR}-210$ & PBMC & HF & Endo et al ${ }^{[2]}(2013)$ \\
\hline †miR-21, miR-25, miR-92a, miR-106b, miR-126, miR-451, miR-590-5p & Plasma & AP, UA & Ren et al ${ }^{[73]}(2013)$ \\
\hline$\leftrightarrow \mathrm{miR}-1, \mathrm{miR}-208 \mathrm{a}, \mathrm{miR}-423-5 \mathrm{p}$ & Plasma & AMI, CAD & Nabialek et al ${ }^{[7]]}(2013)$ \\
\hline$\uparrow \mathrm{miR}-30 \mathrm{a}, \mathrm{miR}-210$ & Serum & HF & Zhao et al ${ }^{[5]}(2013)$ \\
\hline $\begin{array}{l}\uparrow \mathrm{miR}-337-5 \mathrm{p}, \mathrm{miR}-433, \mathrm{miR}-485-3 \mathrm{p}, \mathrm{miR}-1, \mathrm{miR}-122, \mathrm{miR}-126, \mathrm{miR}-133 \mathrm{a} / \mathrm{b} \text {, } \\
\mathrm{miR}-199 \mathrm{a}\end{array}$ & Plasma & $\mathrm{AP}, \mathrm{UA}$ & $D^{\prime}$ Alessandra et al ${ }^{[76]}(2013)$ \\
\hline \multicolumn{4}{|l|}{$\leftrightarrow$ miR-17-5p, miR-92a, miR-145, miR-155, miR-208a, miR-375, miR-799-5p } \\
\hline ImiR-103, miR-142-3p, miR-30b, miR-342-3p & Plasma & HF & Ellis et al ${ }^{[7]}(2013)$ \\
\hline$\uparrow \mathrm{miR}-122, \mathrm{miR}-200 \mathrm{~b}, \mathrm{miR}-520 \mathrm{~d}-5 \mathrm{p}, \mathrm{miR}-622$ & WB and & $\mathrm{HF}$ & Vogel et al ${ }^{[73]}(2013)$ \\
\hline ImiR-558 & serum & & \\
\hline$\lceil\mathrm{miR}-21$, miR-133a, miR-423-5p, miR-499-5p & Plasma & HF, NSTEMI & Olivieri et al ${ }^{[0]}(2013)$ \\
\hline \multicolumn{4}{|l|}{$\leftrightarrow \mathrm{miR}-1, \mathrm{miR}-20 \mathrm{a}$} \\
\hline$\uparrow \mathrm{miR}-133 \mathrm{a}$ & Plasma & AMI, AP & Wang et al ${ }^{[00]}(2013)$ \\
\hline ImiR-214 & Plasma & AMI, AP, UA & Lu et al $l^{[01]}(2013)$ \\
\hline
\end{tabular}

AAA: Abdominal aortic aneurysm; ACS: Acute coronary syndrome; AMI: Acute myocardial infarction; AP: Angina pectoris; CAD: Coronary artery disease; CV: Cardiovascular; HF: Heart failure; LVI: Left ventricular ischemia; NSTEMI: Non-ST-elevation myocardial infarction; PBMC: Peripheral blood

\subsection{Targeting Receptors}

\subsubsection{Adenosine receptors}

Adenosine, a purine nucleoside generated extracellularly from adenine nucleotides released by cells as a result of direct stimulation, hypoxia, trauma, or metabolic stress, is a well-known physiologic and pharmacologic agent (Jackson et al., 1996).

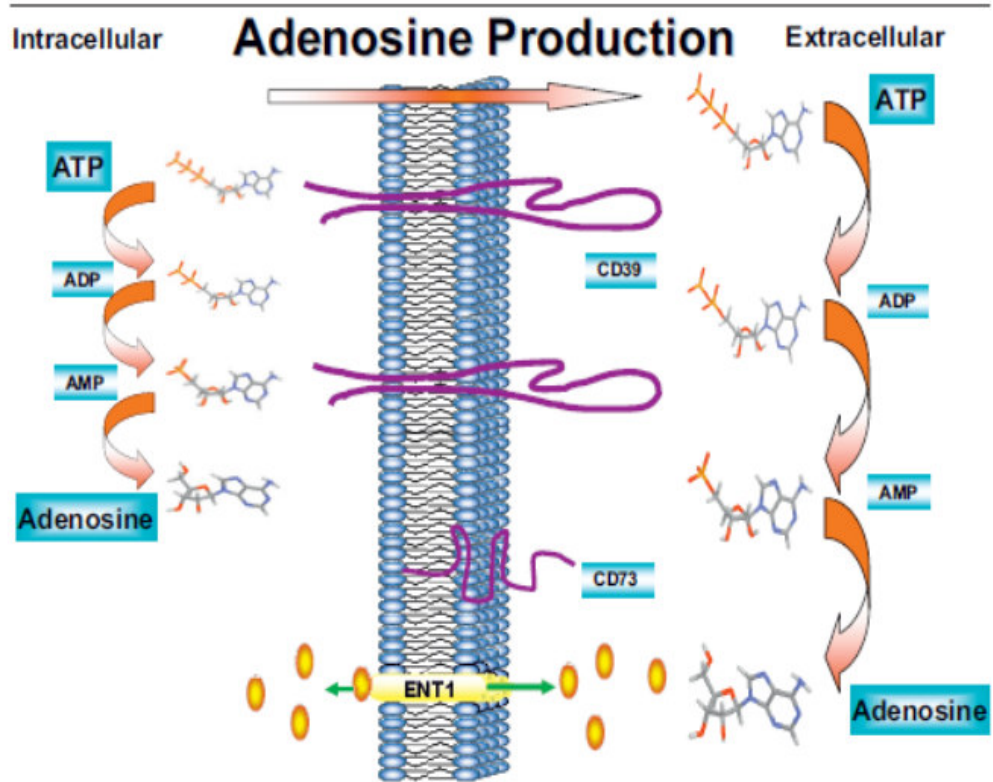

Figure - 2 formation of adenosine from adenine nucleotides (taken from Jackson et al., 1996)

Adenosine is formed both intracellularly and extracellularly from adenine nucleotides, which are sequentially dephosphorylated to adenosine. Intracellular adenosine may be transported into the extracellular space via 
facilitated transport, and extracellular adenosine is also taken up by cells through the same transporter, equilibrative nucleoside transporter 1(ENT1). Two cell surface molecules, CD39 and CD73 (nucleoside triphosphate phosphohydrolase and ecto-50-nucleotidase, respectively), catalyze the dephosphorylation of adenine nucleotides to adenosine in the extracellular space.

Adenosine is synthesized by the cardiac wall and exerts numerous cardioprotective and anti-vaso occlusive actions. The interstitial levels of this nucleoside are elevated in certain pathophysiological conditions (Bruce et al., 2011 and Jackson et al., 1996).

Recent studies demonstrate that adenosine, acting at its receptors, promotes wound healing by stimulating both angiogenesis and matrix production. Subsequently, adenosine and its receptors have also been found to promote fibrosis (excess matrix production) in the skin, lungs, and liver, but to diminish cardiac fibrosis (Headrick et al., 2013).

In addition to a clear role in cardio protection, adenosine exerts a multitude of actions on the physiological regulation of the heart, including coronary vasodilation, heart rate control and AV nodal conduction, angiogenesis, myocardial hypertrophy and remodeling and fibrosis (Bruce et al., 2011 and Headrick et al., 2013).

Exogenous as well as endogenous adenosine inhibits Fetal Calf Serum (FCS)-induced proliferation of CFs (Cardiac Fibroblasts). Because some factors that inhibit cell proliferation also inhibit cellular hypertrophy and ECM synthesis, it was hypothesized that adenosine may be an endogenous factor that attenuates hypertrophy of and collagen synthesis by CFs (Dubey et al., 1997).

Cardiomyocytes, vascular smooth muscle cells, and endothelial cells, both vascular and cardiac, have several metabolic pathways for generating large amounts of adenosine. For example, it has been shown that endothelial cells synthesize adenosine and have an adenine pool that is two to three times greater than that of hepatocytes (Dubey et al., 1997 and Mullane et al., 1995).

Cardiac Fibroblasts (CFs), which constitute $60 \%$ of the total heart cells, can also synthesize adenosine. Therefore, substantial amounts of adenosine are synthesized locally within the cardiac wall, in part by CFs, thus ensuring pharmacologically active levels of adenosine in the heart (Dubey et al., 1998).

The AR family is comprised of four Class - A G protein coupled receptors (GPCRs), the A1, A2A, A2B and A3 ARs. They exert distinct pharmacological actions through differential coupling to intracellular $\mathrm{G}$ proteins; the $\mathrm{A} 1 \mathrm{AR}$ and $\mathrm{A} 3 \mathrm{AR}$ preferentially activate $\mathrm{Gi}=\mathrm{o}$ proteins to inhibit adenylyl cyclase activity and subsequent cAMP production, while the $\mathrm{A} 2 \mathrm{~A}$ AR and $\mathrm{A} 2 \mathrm{~B}$ AR preferentially stimulate Gs proteins to activate adenylyl cyclase activity and increase cAMP accumulation. The A2B AR has also been shown to stimulate robust Gq=11 protein activation in some cell types. ARs, and the A2B AR in particular, have also been shown to couple to additional transmembrane and intracellular proteins, which may influence downstream signal transduction (Fredholm et al., 2001 and Sun et al., 2016).

All four ARs are expressed in the heart and synchronous activation of multiple subtypes' results in both complementary and opposing signal transduction for the fine tuned regulation of cardiac function. Interestingly, both pro- and anti-fibrotic actions have been attributed to AR activation. (Bruce et al., 2011, Chan et al., 2009 and Karmouty et al., 2013) to date, the preponderance of evidence has implicated the A2B AR in cardiac fibrosis (Novitskaya et al., 2016).

A number of studies demonstrate that stimulated adenosine receptors play different roles in the pathogenesis of fibrosis depending on the tissue. Adenosine A2B receptors in the heart inhibit fibrosis, whereas these same receptors promote fibrosis in the lungs. In contrast, in the skin, liver, and lungs, adenosine A2A and A2B receptors both mediate an increase in fibrosis. It is also striking that different adenosine receptors appear to play a dominant role in fibrosis in different organs; adenosine A2A receptors are the dominant receptors in the skin, peritoneum, and liver but A2B receptors are responsible for pulmonary fibrosis (Bruce et al., 2011 and Vecchio et al., 2017).

Adenosine has been implicated in cardioprotective events, notably in the context of cardiac hypertrophy. Its levels are elevated in cardiac hypertrophy, experimental hypertension, acute and chronic phases of myocardial infarction and during the progression of heart failure. Adenosine exerts anti-hypertrophic and anti-adrenergic effects and is proposed to be cardioprotective in the setting of heart failure (S-L Puhl et al., 2016).

Growth-arrested confluent cardiac fibroblast monolayers were stimulated with $2.5 \%$ fetal calf serum (FCS) in the presence and absence of adenosine, 2-chloroadenosine (stable adenosine analogue), or modulators of adenosine levels including (1) erythro-9-(2-hydroxy-3-nonyl) adenine (adenosine deaminase inhibitor), (2) dipyridamole (adenosine transport blocker), and (3) iodotubericidin (adenosine kinase inhibitor) (Dubey et al., 1998).

All agents inhibited in a concentration-dependent fashion FCS-induced [3H] proline and [3H] leucine incorporation. These effects were blocked by KF17837 (selective A2 antagonist) and 1,3-dipropyl-8-(psulfophenyl) xanthine (A1/A2 receptor antagonist) but not by 8-cyclopentyl-1,3-dipropylxanthine (selective A1 antagonist), thus excluding the participation of A1 receptors. The lack of effect of CGS21680 (selective A2A agonist) excluded involvement of $\mathrm{A} 2 \mathrm{~A}$ receptors, thus suggesting a major role for $\mathrm{A} 2 \mathrm{~B}$ receptors. Comparisons of the inhibitory potencies of $N 6$-cyclopentyladenosine (selective A1 agonist), 59- $N$-ethylcarboxamidoadenosine (A1/A2 agonist), 
and 59- $N$-methylcarboxamidoadenosine (A1/A2 agonist) were consistent with that of an A2B receptor subtype mediating the inhibitory effects. It is concluded that adenosine inhibits FCS-induced collagen and total protein synthesis in cardiac fibroblasts via activation of A2B receptors. These studies suggest, but do not prove, that endogenous adenosine may protect against cardiac fibrosis (Bruce et al., 2011, Dubey et al., 1997 and Dubey et al., 1998).

Dubey and colleagues have reported that adenosine A2B receptor activation inhibits cardiac fibroblast production of collagen in vitro. In agreement with these original findings, Wakeno and colleagues have reported that adenosine A2B receptor stimulation diminishes fibrosis and remodeling of the myocardium after infarction (Dubey et al., 1998 and Wakeno et al., 2006).

Among the four subtypes of adenosine receptors (AdoRs), the A2B AdoR was expressed at the highest level in human cardiac fibroblasts (HCF). NECA, a stable analog of adenosine, significantly increased the release of IL6 in a concentration-dependent manner. In addition, NECA increased the expression of a-smooth muscle actin and a-1 pro-collagen, and the production of collagen from HCF. The effects of NECA on release of IL-6, collagen, ST-2, and PAPPA and expression of a-smooth muscle actin and a-1 pro-collagen were completely abolished by a selective A2B AdoR antagonist, GS-6201 (Wakeno et al., 2006).

A2B AdoR is the predominant subtype of AdoRs expressed in primary human HCF. Activation of this receptor increases the release of IL-6, production of collagen, expression of fibrotic markers and release of biomarkers of CVD. These findings suggest that A2B AdoR might mediate the fibrotic response in heart diseases (Hongyan et al., 2010).

Deletion of $5^{\prime}$ ectonucleotidase, one of the main regulators of adenosine metabolism, and the subsequent reduction in adenosine levels led to pronounced cardiac hypertrophy in a rat model of experimental hypertension, while stimulation of adenosine receptors attenuated cardiomyocyte hypertrophy in this model (Wakeno et al., 2006 and $\mathrm{Xu}$ et al., 2008).

$\mathrm{Xu}$ et al presented that activation of adenosine A1 receptors prevented phenylephrine-mediated cardiomyocyte hypertrophy and cardiac fibrosis in a model of compensated hypertrophy and that this cardioprotection is accompanied by up-regulation of the A1 receptors. These data suggest that full or partial agonists at adenosine A1 receptors may also mitigate cardiac remodeling and thus potentially dysfunction in response to $\alpha 1$-adrenoceptor stimulation (Xu et al., 2008).

Besides its anti-hypertrophic effects, adenosine mediates anti-fibrotic effects through adenosine A2B receptors in vitro and in vivo. Furthermore, it is previously observed that adenosine prevents the expression of the pro-inflammatory cytokine TNF- $\alpha$ in the rat and failing human heart (Epperson et al., 2009).

\subsection{Targeting enzymes}

\subsubsection{Lysyl Oxidase like 2 (LOXL2)}

Interstitial fibrosis plays a key role in the development and progression of heart failure. An enzyme that cross links collagen Lysyl Oxidase-like 2 (Lox12) is essential for interstitial fibrosis and mechanical dysfunction of pathologically stressed hearts. In mice, cardiac stress activates fibroblasts to express and secrete Loxl2 into the interstitium, triggering fibrosis, systolic and diastolic dysfunction of stressed hearts. In diseased human hearts, LOXL2 is upregulated in cardiac interstitium; its levels correlate with collagen crosslinking and cardiac dysfunction. LOXL2 is also elevated in the serum of heart failure (HF) patients, correlating with other HF biomarkers, suggesting a conserved LOXL2-mediated mechanism of human HF (Jin Yang et al.,2016).

Lysyl oxidase-like 2 (LOXL2) is upregulated in the interstitium of diseased mouse and human hearts. Increased LOXL2 expression leads to increased TGF- $\beta 2$ production, triggering the formation and migration of myofibroblasts with enhanced collagen deposition and crosslinking in the hypertrophic regions of stressed hearts. These effects result in interstitial fibrosis and cardiac dysfunction (Jin Yang et al., 2016).

In mechanically stressed hearts, LOXL2 expression is activated in the fibroblasts, which then release LOXL2 proteins into the interstitial space, particularly in the hypertrophic area where the mechanical stress is highest. This suggests that LOXL2 activation is a response of cardiac fibroblasts to enhanced mechanical stress to maintain structural integrity of the heart. Indeed, LOXL2 elevation has multiple biological effects that promote interstitial collagen formation. LOXL2 not only triggers myofibroblast transformation to enhance collagen production (through PI3K-AKT-mTOR and TGF-b), but also augments collagen strength (by crosslinking collagen fibers). LOXL2 also stimulates myofibroblasts migration to large areas of the heart, where these cells produce collagen fibers. Collagen fibers, once released into the interstitial space, are crosslinked by LOXL2 to form bundles of collagen that are much stiffer than isolated collagen fibers. Furthermore, activated fibroblasts secrete more LOXL2 and collagen, creating a positive feedback loop to sustain the fibrotic process. All these factors, in combination, trigger diffuse interstitial fibrosis of pathologically stressed hearts (Jin Yang et al., 2016 and Davis et al., 2014).

In patients with HF, LOXL2 levels are elevated in heart tissues and serum, its levels correlating with cardiac dysfunction and HF biomarker levels. In mice, LOXL2 activation is essential for cardiac fibrosis and HF development. The genetic or pharmacological inhibition of LOXL2 greatly reduces cardiac fibrosis and halts HF 
progression. The human sample and animal efficacy studies, in combination, suggest a pathogenic role of LOXL2 in cardiac fibrosis and human HF. Our studies therefore delineate a novel LOXL2-mediated HF mechanism and provide new insights into HF therapy (Davis et al., 2014, Kong et al., 2014 and Schelbert et al., 2015).

The LOXL2-mediated stress reaction, although capable of reinforcing tissue strength in the face of increased mechanical stress, is ultimately maladaptive. Excessive amount of collagen fibers in cardiac interstitial space impede coronary vasodilation, oxygen diffusion and electromechanical coordination between cardiomyocytes, causing contractile abnormalities (systolic pump dysfunction). Meanwhile, the increase of collagen and its crosslinking stiffens the left ventricle, impairing ventricular relaxation and filling (diastolic pump dysfunction. Therefore, the LOXL2-mediated interstitial reaction leads to both systolic and diastolic abnormalities, revealing a novel therapeutic avenue for HF. The diastolic aspect of LOXL2 effects is particularly important, given the recognition and increasing prevalence of diastolic dysfunction as part of HF syndrome and the lack of approved therapy for HFpEF with primarily diastolic failure (Kasner et al., 2011).

Although LOXL2 can interact with TGF-b in models of cancer and bone remodeling, the roles of LOXL2 gene in HF have not been demonstrated in mouse genetic models in vivo. By generating a new LOXL2 genetic model with fibroblast-specific LOXL2 knockout, we showed crucial roles of LOXL2 in cardiac fibroblasts for stress-induced interstitial fibrosis and cardiac dysfunction. What remains unanswered is how LOXL2-mediated changes of extracellular collagen composition affect TGF-b processing and signaling and guide the cellular process of myofibroblast migration. Given that LOXL2 is transcriptionally activated in the stressed hearts, it will be essential to know what factors control the expression of LOXL2 gene in the hearts. Gene expression regulation can occur at the chromatin, transcription or posttranscriptional level, mediated respectively by epigenetic factors (chromatin-regulating factors, long noncoding RNAs), transcription factors and MicroRNAs. Understanding LOXL2 regulation will provide an opportunity to integrate cardiac fibrosis with epigenetics and RNA mechanisms of HF and to identify additional new targets for HF therapy (Atsawasunan et al., 2008).

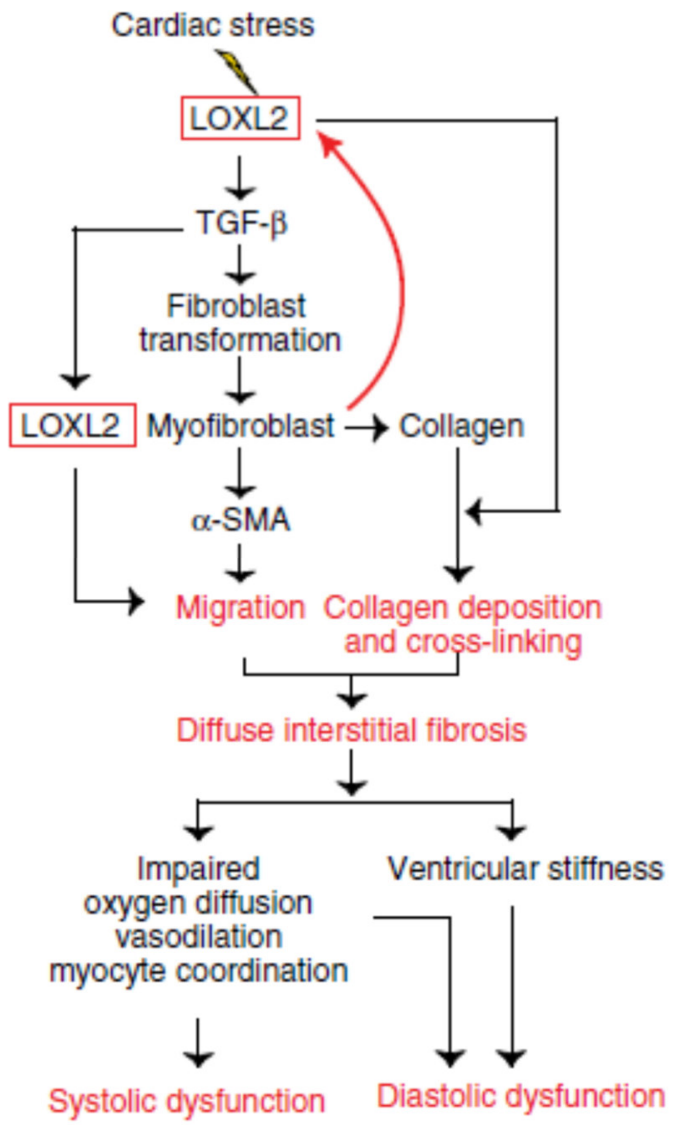

Figure 3 - a working model of how cardiac stress activates LOXL2 to trigger myofibroblast transformation, collagen synthesis, collagen crosslinking and myofibroblast migration, leading to diffuse ventricular fibrosis and dysfunction. (From Jin Yang et al., 2016)

\subsection{Targeting Oxidative stress}

\subsubsection{N-Acetylcysteine}

Oxidative stress can contribute to the development of cardiac remodeling and fibrosis and these cardiac adaptations 
can ultimately lead to failing of the myocardium. For example, oxidative stress has been demonstrated to increase the expression of cardiac collagen types I and IV and fibronectin and impair cardiac contractility in diabetic rats (Tsutsui et al., 2011).

There is also evidence that oxidative stress plays a role in the development of angiotensin II-dependent cardiac fibrosis in rats (M-E Worou et al., 2013) and mice (Li et al., 2013).

Reducing oxidative stress can minimize cardiac remodeling in the setting of HF. Cardiac-specific over expression of MST-1 induces apoptosis leading to cardiac remodeling (Yamamoto et al., 2003).

Importantly, there is evidence that oxidative stress can induce apoptosis which provides a basis to target oxidative stress to reduce apoptosis-induced cardiac remodeling (Kumar et al., 2002).

NAC can attenuate cardiac fibrosis and remodeling in the setting of HF. As these factors play major pathological roles in the development of HF (Beverly et al., 2016). It has previously been shown that NAC has anti-fibrotic effects in experimental hypertrophic cardiomyopathy (Lombardi et al., 2009).

There is evidence that left ventricular glutathione content is reduced in $\mathrm{HF}$, and of particular interest, restoring the glutathione content via oral supplementation of its precursor, N-Acetylcysteine (NAC), reduced oxidative stress, and restored HF-related cardiac damage and function in rats. In addition to reducing oxidative stress, NAC treatment reduced the expression of the pro-inflammatory cytokine tumor necrosis factor alpha (TNF- $\alpha$ ) and its receptor expression in these rats (Adamy et al., 2007).

Beverly et al discover two major novel findings in their study which aimed at assessing the role of NAC in reducing cardiac fibrosis and remodeling. First, cardiac perivascular and interstitial fibrosis and cardiac expression of collagen types I and III were greater in MST-1 mice compared to WT mice. Of interest, they found that perivascular and cardiac interstitial fibrosis were less in MST-1 mice treated with NAC compared to those treated with saline vehicle. Consistent with a reduction in cardiac fibrosis, cardiac collagen types I and III expression was also less in NAC-treated MST-1 mice than those treated with saline vehicle. These data provide direct evidence that NAC can prevent the development of cardiac fibrosis in the setting of HF. Together, these findings indicate that NAC can prevent the development of cardiac fibrosis in a mouse model of dilated cardiomyopathy. Secondly, it was found that the thickness of the interventricular septum was greater in MST-1 mice compared to WT mice. In NAC-treated MST-1 mice, the thickness of the interventricular septum was less compared to vehicle treated MST-1 mice. These findings indicate that NAC treatment can attenuate HF-dependent cardiac remodeling (Beverly et al., 2016 and A-H Talasaz et al., 2013).

NAC treatment reduced serum levels of TNF-a, transforming growth factor $\beta$ (TGF- $\beta$ ), matrix metalloproteinase 9 (MMP-9), and MMP-2 in patients with acute myocardial infarction (A-H Talasaz et al., 2013). These cytokines and enzymes play an important role in the development of cardiac fibrosis, remodeling, and subsequent cardiac dysfunction (Skyschally et al., 2007).

Therefore, NAC is likely to simultaneously target multiple mechanisms which underpin the progressive worsening of cardiac function in HF. In particular, cardiac fibrosis remains an independent risk factor for cardiomyopathy-related morbidity and mortality (Leyva et al., 2012 and Gulati et al., 2013) and treatment interventions that effectively target cardiac fibrosis remain an unmet clinical need.

There is evidence that perivascular fibrosis is associated with reduced coronary perfusion in patients with nonischemic HF. As impaired coronary perfusion can in turn have a direct impact on cardiac function, these findings provide a basis for targeting perivascular fibrosis in the treatment of HF. Beverly et al found that perivascular fibrosis was less in MST-1 mice treated with NAC compared to those treated with saline. Thus, the findings provide evidence that NAC can reduce cardiac perivascular and interstitial fibrosis associated with $\mathrm{HF}$ (Beverly et al., 2016 and Z Dai et al., 2012).

It is also of interest to note that NAC can reduce oxidative stress, improve cardiac index, and reduce mortality in patients with acute myocardial infarction (Simon et al., 1996). Overall, these data provide evidence that NAC can provide cardioprotective effects to HF patients potentially via attenuation of cardiac fibrosis (Lombardi et al., 2009 and Marian et al., 2006).

\section{Conclusions}

Despite the presence of different groups of drugs for treating cardiac fibrosis such as antihypertensive agents, antiinflammatory agents and heart rate reducing agents; the prevalence of cardiovascular diseases is increasing. Appreciably ample of novel therapeutic targets are being considered and few are under clinical trials; the effectiveness of which brings us with a green light as a preventive tool of cardiac fibrosis and as an indirect tool for prevention of heart failure and myocardial infarction. More importantly the implementation of epigenetic based therapies such as MicroRNAs is one of the first miracles in this aspect keeping in mind that few agents which were previously indicated for treatment of other medical conditions are nowadays reshuffled for the management of cardiac fibrosis. 


\section{Future perspectives}

There are tenths of new treatment targets for cardiac fibrosis that are under active clinical trials. It is believed that these newer targets can effectively battle against cardiac fibrosis in order to reduce the morbidity and mortality of cardiovascular diseases. In addition, this review discovered existing agents indicated for medical conditions other than cardiac fibrosis, as potential targets of therapy such as NSAIDs. These agents can be used effectively as alternatives until the newer agents come into existence.

Furthermore newer targets, those targeting sodium potassium ATPase, Peroxisome Proliferator activated receptor $\mathrm{V}$ and Epoxide hydroxylase enzyme are on the way representing signaling pathways, receptors and enzymes respectively and hence increasing the probability that our world might be cardiovascular disease free in the near future.

\section{References}

Adamy, C., P. Mulder, L. Khouzami, N. Andrieu-abadie, N. Defer, G. Candiani, et al. 2007. Neutral sphingomyelinase inhibition participates to the benefits of $\mathrm{N}$-acetylcysteine treatment in post-myocardial infarction failing heart rats. J. Mol. Cell. Cardiol. 43:344-353[CrossRef]

Adebiyi OA, Adebiyi OO, Owira PMO (2016) Naringin Reduces Hyperglycemia-Induced Cardiac Fibrosis by Relieving Oxidative Stress. PLoS ONE 11(3): e0149890. doi:10.1371/journal. pone.0149890

Anderson KR, Sutton MG, Lie JT (1979). Histopathological types of cardiac fibrosis in myocardial disease. J Pathol, 128:79-85.[CrossRef][wiley online library]

Anna Biernacka and Nikolaos G Frangogiannis. Aging and Cardiac Fibrosis, Aging and Disease • Volume 2, Number 2, April 2011

Atsawasuwan, P. et al. Lysyl oxidase binds transforming growth factor-beta and regulates its signaling via amine oxidase activity. J. Biol. Chem. 283, 34229-34240 (2008)

Aurora AB, Mahmoud AI, Luo X et al. Microrna-214 protects the mouse heart from ischemic injury by controlling ca (2) (+) overload and cell death. J. Clin. Invest. 122(4), 1222-1232 (2012)

Azibani F, Benard L, Schlossarek S et al. Aldosterone inhibits antifibrotic factors in mouse hypertensive heart. Hypertension 59(6), 1179-1187 (2012)

Bartholomeu JB, Vanzelli AS, Rolim NP et al. Intracellular mechanisms of specific beta-adrenoceptor antagonists involved in improved cardiac function and survival in a genetic model of heart failure. J. Mol. Cell. Cardiol. 45(2), 240-249 (2008)

Baudino TA, Carver W, Giles W, Borg TK (2006) Cardiac fibroblasts: friend or foe. Am J Physiol Heart CircPhysiol 291: 1015-1026

Bauersachs J. Regulation of myocardial fibrosis by MicroRNAs. J CardiovascPharmacol. 2010;56(5):454-459

Becher PM, Lindner D, Miteva K et al. Role of heart rate reduction in the prevention of experimental heart failure: Comparison between If-channel blockade and beta-receptor blockade. Hypertension 59(5), 949-957 (2012)

Beverly Giam, Po-Yin Chu, SanjayaKuruppu, A. Ian Smith, Duncan Horlock, Helen Kiriazis, Xiao-Jun Du, David M. Kaye \&Niwanthi W. Rajapakse . N-acetylcysteine attenuates the development of cardiac fibrosis and remodeling in a mouse model of heart failure, 2016 | Vol. 4 | Iss. 7 | e12757)

Boon RA, Seeger T, Heydt S, Fischer A, Hergenreider E, HorrevoetsAJ,Vinciguerra M, Rosenthal N, Sciacca S, Pilato M, et al (2011) MicroRNA-29 in aortic dilation: implications for aneurysm formation. Circ Res 109: 1115- 1119

Brilla CG, Funck RC, Rupp H. Lisinopril-mediated regression of myocardial fibrosis in patients with hypertensive heart disease. Circulation 102(12), 1388-1393 (2000)

Brilla CG, Matsubara L, Weber KT. Advanced hypertensive heart disease in spontaneously hypertensive rats. Lisinopril-mediated regression of myocardial fibrosis. Hypertension 28(2), 269-275 (1996).

Brilla CG, Zhou G, Matsubara L, et al. Collagen metabolism in cultured adult rat cardiac fibroblasts: response to angiotensin II and aldosterone. J Mol Cell Cardiol. 1994;26:809-820[CrossRef]

Brilla CG. Regression of myocardial fibrosis in hypertensive heart disease: Diverse effects of various antihypertensive drugs. Cardiovasc. Res. 46(2), 324-331 (2000).

Brooks WW, Conrad CH (2000). Myocardial fibrosis in transforming growth factor beta (1) heterozygous mice. J Mol Cell Cardiol, 32:187-95[CrossRef]

Bruce N. Cronstein. Adenosine receptors and fibrosis: a translational review F1000 Biology Reports 2011, 3:21 (doi:10.3410/B3-21

Bujak M, Frangogiannis NG (2007). The role of TGF-beta signaling in myocardial infarction and cardiac remodeling. Cardiovasc Res, 74:184-95

Busseuil D, Shi Y, Mecteau M et al. Heart rate reduction by ivabradine reduces diastolic dysfunction and cardiac fibrosis. Cardiology 117(3), 234-242 (2010)

Chan, E. S. L., and Cronstein, B. N. (2009). Adenosine in fibrosis. Mod. Rheumatol. 20, 114-122. doi: $10.1007 / \mathrm{s} 10165-009-0251-4$ 
Chen S, Evans T, Mukherjee K, Karmazyn M, Chakrabarti S. Diabetes induced myocardial structural changes: role of endothelin-1 and its receptors. J Mol Cell Cardiol. 2000; 32:1621-1629. doi: 10.1006/ jmcc.2000.1197. [CrossRef]

Claudio Napoli, Vincenzo Grimaldi, Maria Rosaria De Pascale, Linda Sommese, Teresa Infante, Andrea Soricelli. Novel epigenetic-based therapies useful in cardiovascular Medicine, World J Cardiol2016 February 26; 8(2): 211-219)

Da Costa Martins PA, Salic K, Gladka MM, Armand AS, Leptidis S, el Azzouzi H, Hansen A, Coenen-de Roo CJ, Bierhuizen MF, van der Nagel R, et al (2010) MicroRNA-199b targets the nuclear kinase Dyrk1a in an autoamplification loop promoting calcineurin/NFAT signalling. Nat Cell Biol 12: 1220-1227[CrossRef][Pubmed]

Dai, Z., T. Aoki, Y. Fukumoto, and H. Shimokawa. 2012. Coronary perivascular fibrosis is associated with impairment of coronary blood flow in patients with non-ischemic heart failure. J. Cardiol. 60:416-421

Dashwood MR, Abraham D. Endothelin: from bench to bedside and back. Pharmacol Res. 2011; 63:445-447. doi: 10.1016/j.phrs.2011.04.005

Davis, J. \&Molkentin, J. D. Myofibroblasts: trust your heart and let fate decide. J. Mol. Cell. Cardiol. 70, 9-18 (2014)

De Boer RA, Yu L, van Veldhuisen DJ. Galectin-3 in cardiac remodeling and heart failure. Curr. Heart Fail. Rep. $7(1), 1-8(2010)$

Dobaczewski M, Chen W, Frangogiannis NG (2011) Transforming growth factor (TGF) $\beta$ signaling in cardiac remodeling. J Mol Cell Cardiol 51: 600-606

Dubey RK, Gillespie DG, Jackson EK: Adenosine inhibits collagen and protein synthesis in cardiac fibroblasts: role of A2B receptors. Hypertension 1998, 31:943-8

Dubey RK, Gillespie DG, Mi Z, Jackson EK. Exogenous and endogenous adenosine inhibits fetal calf seruminduced growth of cardiac fibroblast:possible role of A2B receptors. Circulation. 1997; 96:2656 -2666.) [CrossRef]

Duerrschmid C, Crawford JR, Reineke E et al. TNF receptor 1 signaling is critically involved in mediating angiotensin-II-induced cardiac fibrosis. J. Mol. Cell. Cardiol. 57, 59-67 (2013)

Duisters, R.F.; Tijsen, A.J.; Schroen, B.; Leenders, J.J.; Lentink, V.; van der Made, I.; Herias, V.; van Leeuwen, R.E.; Schellings, M.W.; Barenbrug, P.; et al. miR-133 and miR-30 regulate connective tissue growth factor: Implications for a role of microRNAs in myocardial matrix remodeling. Circ. Res. 2009, 104,170-178.

Duygu B, Poels EM, da Costa Martins PA. Genetics and epigenetics of arrhythmia and heart failure. Front Genet 2013; 4: 219 [PMID: 24198825 DOI: 10.3389/f gene. 2013. 00219])

Eline L. Vegter1, Peter van der Meer1, Leon J. deWindt2, Yigal M. Pinto3, and Adriaan A. Voors1. MicroRNAs in heart failure: from biomarker to target for therapy, review, European Journal of Heart Failure (C) 2016 European Society of Cardiology

Epperson SA, Brunton LL, Ramirez-Sanchez I, Villarreal F (2009). Adenosine receptors and second messenger signaling pathways in rat cardiac fibroblasts. Am J Physiol Cell Physiol 296: C1171-C1177

Euler G (2015) Good and bad sides of TGF $\beta$-signaling in myocardial infarction. Front Physiol 6: 66

Francois Roubille, David Busseuil, NolwennMerlet, Ekaterini A Kritikou, Eric Rhe'aume, Jean-Claude Tardif. Investigational drugs targeting cardiac fibrosis, Expert Rev. Cardiovasc. Ther. Early online, 1-15 (2014)

Fredholm, B. B., IJzerman, A. P., Jacobson, K. A., Klotz, K. N., and Linden, J. (2001). Nomenclature and classification of adenosine receptors, International Union of Pharmacology. XXV..Pharmacol. Rev. 53, 527552[CrossRef]

Gulati, A., A. Jabbour, T. F. Ismail, K. Guha, J. Khwaja, S. Raza, et al. 2013. Association of fibrosis with mortality and sudden cardiac death in patients with nonischemic dilated cardiomyopathy. JAMA 309:896-908

Hafizi S, Wharton J, Chester AH, Yacoub MH. Profibrotic effects of endothelin-1 via the ETA receptor in cultured human cardiac fibroblasts. Cell PhysiolBiochem. 2004; 14:285-292. doi: 10.1159/000080338

Headrick, J. P., Ashton, K. J., Rose'Meyer, R. B., and Peart, J. N. (2013). Cardiovascular adenosine receptors: expression, actions and interactions. Pharmacol. Ther. 140, 92-111. doi: 10.1016/ j.pharmthera.2013.06.002

HongyanZhong, LuizBelardinelli, Dewan Zeng. Pro-Fibrotic Role of the A2B Adenosine Receptor in Human Cardiac Fibroblasts, 15th annual scientific meeting HFSA S65

Hrayr S. Karagueuzian. Targeting cardiac fibrosis: a new frontier in antiarrhythmic therapy?, Am J Cardiovasc Dis $2011 ; 1(2): 101-109$

Ieki K, Yazaki Y, Yamaoki K et al. Effect of long-term treatment with beta-blocker on cardiac hypertrophy in shr. J. Mol. Cell. Cardiol. 21(Suppl. 5), 113-119 (1989).[CrossRef]

Jackson EK, Koehler M, Mi Z, Dubey RK, Tofovic SP, Carcillo JA, Jones GS. Possible role of adenosine deaminase in vaso-occlusive diseases. J Hypertens. 1996;14:19-29[CrossRef]

Jalil JE, Doering CW, Janicki JS, Pick R, Shroff SG, Weber KT. Fibrillar collagen and myocardial stiffness in the intact hypertrophied rat left ventricle. Circ. Res. 64(6), 1041-1050 (1989)

Jenny Y. Y. Oo, Bianca C. Bernardo, Julie R. McMullen. Therapeutic potential of targeting microRNAs to regulate 
cardiac fibrosis: miR-433 a new fibrotic player, commentary,AnnTransl Med 2016;4(24):548

Jin Yang, Konstantinos Savvatis, Jong Seok Kang, Peidong Fan, Hongyan Zhong, Karen Schwartz, Vivian Barry, Amanda Mikels-Vigdal, Serge Karpinski, DmytroKornyeyev, Joanne Adamkewicz, Xuhui Feng, Qiong Zhou1, Ching Shang, Praveen Kumar, Dillon Phan, Mario Kasner, Begon aLo'pez, Javier Diez, Keith C. Wright, Roxanne L. Kovacs, Peng-Sheng Chen, Thomas Quertermous, Victoria Smith, Lina Yao, CarstenTscho"pe\&Ching-Pin Chang, Targeting LOXL2 for cardiac interstitial fibrosis and heart failure treatment, NATURE COMMUNICATIONS | 7:13710 | DOI: $10.1038 /$ ncomms 13710 |www.nature.com/naturecommunications

Karmouty-Quintana, H., Xia, Y., and Blackburn, M. R. (2013). Adenosine signaling during acute and chronic disease states. J Mol. Med. 91, 173-181. doi: 10.1007/ s00109-013-0997-1

Kasner, M. et al. Diastolic tissue Doppler indexes correlate with the degree of collagen expression and crosslinking in heart failure and normal ejection fraction. J. Am. Coll. Cardiol. 57, 977-985 (2011).

Katwa LC. Cardiac myofibroblasts isolated from the site of myocardial infarction express endothelin de novo. Am J Physiol Heart Circ Physiol. 2003;285:H1132-H1139. doi: 10.1152/ajpheart.01141.2002

Kawamura M, Ito H, Onuki T et al. Candesartan decreases type III procollagen-n-peptide levels and inflammatory marker levels and maintains sinus rhythm in patients with atrial fibrillation. J. Cardiovasc. Pharmacol. 55(5), 511-517 (2010)

Khoueiry Z, Roubille C, Nagot $\mathrm{N}$ et al. Could heart rate play a role in pericardial inflammation? Med. Hypotheses 79(4), 512-515 (2012)

Kong, P., Christia, P. \&Frangogiannis, N. G. The pathogenesis of cardiac fibrosis. Cell. Mol. Life Sci. 71, 549574 (2014)

Kosmala W, Przewlocka-Kosmala M, Szczepanik-Osadnik H, Mysiak A, O’Moore-Sullivan T, Marwick TH. A randomized study of the beneficial effects of aldosterone antagonism on LV function, structure, and fibrosis markers in metabolic syndrome. JACC Cardiovasc. Imaging 4(12), 1239-1249 (2011).)

Kumar, D., H. Lou, and P. K. Singal. 2002. Oxidative stress and apoptosis in heart dysfunction. Herz 27:662$668[$ CrossRef $]$

Kumarswamy R, Volkmann I, Jazbutyte V, Dangwal S, Park DH, ThumT.Transforming growth factor-betainduced endothelial-to-mesenchymal transition is partly mediated by microRNA-21. ArteriosclerThrombVascBiol2012; 32:361-369[CrossRef][Pubmed]

Lakatta EG (2003). Arterial and cardiac aging: major shareholders in cardiovascular disease enterprises: Part III: cellular and molecular clues to heart and arterial aging. Circulation, 107:490-7[CrossRef]

Leask A. Getting to the heart of the matter: new insights into cardiac fibrosis. Circ Res. 2015;116:1269-76

Leyva, F., R. J. Taylor, P. W. Foley, F. Umar, L. J. Mulligan, K. Patel, et al. 2012. Left ventricular midwall fibrosis as a predictor of mortality and morbidity after cardiac resynchronization therapy in patients with nonischemic cardiomyopathy. J. Am. Coll. Cardiol. 60:1659-1667

Li YC, Luo Q, Ge LS et al. Ivabradine inhibits the production of proinflammatory cytokines and inducible nitric oxide synthase in acute coxsackievirus B3-induced myocarditis. Biochem. Biophys. Res. Commun. 431(3), 450-455 (2013)

Li, Y. Q., X. B. Li, S. J. Guo, S. L. Chu, P. J. Gao, D. L. Zhu, et al. 2013. Apocynin attenuates oxidative stress and cardiac fibrosis in angiotensin II-induced cardiac diastolic dysfunction in mice. ActaPharmacol. Sin. $34: 352-359$

Liang H, Zhang C, Ban T, Liu Y, Mei L, Piao X, Zhao D, Lu Y, Chu W, Yang B. A novel reciprocal loop between microRNA-21 and TGF betaRIII is involved in cardiac fibrosis. Int J Biochem Cell Biol2012;44:21522160[CrossRef][Medline]

Lijnen PJ, Petrov VV, Fagard RH (2000) Induction of cardiac fibrosis by angiotensin II. Methods Find ExpClinPharmacol 22: 709-723[CrossRef][Pubmed]

Liu G, Friggeri A, Yang Y, Milosevic J, Ding Q, Thannickal VJ, Kaminski N, Abraham E (2010) MiR-21 mediates fibrogenic activation of pulmonary fibroblasts and lung fibrosis. J Exp Med 207: 1589-1597

Lombardi, R., G. Rodriguez, S. N. Chen, C. M. Ripplinger, W. Li, J. Chen, et al. 2009. Resolution of established cardiac hypertrophy and fibrosis and prevention of systolic dysfunction in a transgenic rabbit model of human cardiomyopathy through thiol-sensitive mechanisms. Circulation 119:1398-1407

Lopez B, Querejeta R, Varo N et al. Usefulness of serum carboxy-terminal propeptide of procollagen type I in assessment of the cardioreparative ability of antihypertensive treatment in hypertensive patients. Circulation 104(3), 286-291 (2001).

Lorenzen J, Kumarswamy R, Dangwal S, Thum T. MicroRNAs in diabetes and diabetes-associated complications. RNA Biol. June 1, 2012. doi:10.4161/rna.20162. http://www.landesbioscience.com /journals/rnabiology/ article/ 20162/. Accessed July 20, 2012[CrossRef][Pubmed]

M Yoshiyama, Y Nakamura, T Omura, Y Izumi, R Matsumoto, S Oda, K Takeuchi, S Kim, H Iwao, J Yoshikawa. Angiotensin converting enzyme inhibitor prevents left ventricular remodelling after myocardial infarction in 
angiotensin II type 1 receptor knockout mice, Heart 2005;91:1080-1085

Mak GJ, Ledwidge MT, Watson CJ et al. Natural history of markers of collagen turnover in patients with early diastolic dysfunction and impact of eplerenone. J. Am. Coll. Cardiol. 54(18), 1674-1682 (2009)

Maleszewska M, Moonen JR, Huijkman N, van de Sluis B, Krenning G, Harmsen MC. IL-1beta and TGFbeta2 synergistically induce endothelial to mesenchymal transition in an NFkappaB-dependent manner. Immunobiology. 2013;218:443-54[CrossRef][Pubmed]

Mandel WJ, Chen PS, Weiss JN and Karagueuzian HS. Glycolytic Inhibition Causes Spontaneous Ventricular Fibrillation in Aged Hearts. Am J Physiol Heart CircPhysiol 2011

Marian, A. J., V. Senthil, S. N. Chen, and R. Lombardi. 2006. Antifibrotic effects of antioxidant N-acetylcysteine in a mouse model of human hypertrophic cardiomyopathy mutation. J. Am. Coll. Cardiol. 47:827-834.

MariannGyöngyösi, Johannes Winkler, Isbaal Ramos, Quoc-Tuan Do, HüseyinFirat, Kenneth McDonald, Arantxa González, Thomas Thum, Javier Díez, FrédéricJaisser, Anne Pizard, FaiezZannad. Myocardial fibrosis: biomedical research from bench to bedside, European Journal of Heart Failure (2017) 19, 177-191

Matsusaka H, Kinugawa S, Ide T et al. Angiotensin II type 1 receptor blocker attenuates exacerbated left ventricular remodeling and failure in diabetes-associated myocardial infarction. J. Cardiovasc. Pharmacol. 48(3), 95-102 (2006).

Mori L, Bellini A, Stacey MA, Schmidt M, Mattoli S. Fibrocytes contribute to the myofibroblast population in wounded skin and originate from the bone marrow. Exp Cell Res. 2005;304:81-90[CrossRef][Pubmed]

Mullane K, Bullough D. Harnessing an endogenous cardioprotective mechanism: cellular sources and sites of action of adenosine. J MolCellCardiol. 1995;27:1041-1054[CrossRef]

Nade VS, Dharmadhikari PP, Kawale LA. Anti-Fibrotic Effect of Irbesartan via Attenuation of Endoplasmic Reticulum stress in Isoprenaline-Induced Myocardial Fibrosis. Int J Pharmacol and Clin Sci. 2015;4(4):7682.

Nagpal, V.; Rai, R.; Place, A.T.; Murphy, S.B.; Verma, S.K.; Ghosh, A.K.; Vaughan, D.E. MiR-125b is critical for fibroblast-to-myofibroblast transition and cardiac fibrosis. Circulation 2016, 133, 291-301.

Novitskaya, T., Chepurko, E., Covarrubias, R., Novitskiy, S., Ryzhov, S. V., Feoktistov, I., et al. (2016). Extracellular nucleotide regulation and signaling in cardiac fibrosis. J. Mol. Cell. Cardiol. 93, 47-56. doi: 10.1016/j.yjmcc.2016. 02.010

Pan Z, Sun X, Shan H et al. Microrna-101 inhibited postinfarct cardiac fibrosis and improved left ventricular compliance via the fbj osteosarcoma oncogene/transforming growth factor-betal pathway. Circulation 126(7), 840-850 (2012)

Paul Milliez, NoeleenDeAngelis, Catherine Rucker-Martin, Antoine Leenhardt, Eric Vicaut,EstelleRobide, Philippe Beaufils, Claude Delcayre, Ste'phane N. Hatem. Spironolactone reduces fibrosis of dilated atria during heart failure in rats with myocardial infarction, European Heart Journal (2005) 26, 2193-2199

Piera Velazquez S, Li Z, Jimenez SA (2011) Role of endothelial-mesenchymal transition (EndoMT) in the pathogenesis of fibrotic disorders. Am J Pathol 179: 1074-1080.

Porter KE, Turner NA (2009) Cardiac fibroblasts: At the heart of myocardial remodeling. PharmacolTher 123(2):255-278

Prabhu SD, Frangogiannis NG (2016) The Biological Basis for Cardiac Repair After Myocardial Infarction: From Inflammation to Fibrosis. Circ Res 119: 91-11

Quiat D, Olson EN. MicroRNAs in cardiovascular disease: from pathogenesis to prevention and treatment. $J$ Clin Invest. 2013;123(1):11-18

Raghvendra K. Dubey, Delbert G. Gillespie, Edwin K. Jackson. Adenosine Inhibits Collagen and Protein Synthesisin Cardiac Fibroblasts Role of A2B Receptors, Hypertension. 1998;31:943-948

RomainBos, Nathalie Mougenot, Odile Me' diani, Paul M. Vanhoutte, and Philippe Lechat. Potassium Canrenoate, an Aldosterone Receptor Antagonist, Reduces Isoprenaline-Induced Cardiac Fibrosis in the Rat, the journal of pharmacology and experimental therapeutics, JPET 309:1160-1166, 2004, Vol. 309, No. 3)

Rosenkranz S (2004). TGF-betal and angiotensin networking in cardiac remodeling. Cardiovasc Res, 63:42332[CrossRef]

Rosenkranz S, Flesch M, Amann K, Haeuseler C, Kilter H, Seeland U, Schluter KD, Bohm M (2002). Alterations of beta-adrenergic signaling and cardiac hypertrophy in transgenic mice overexpressing TGF-beta (1). Am J Physiol Heart CircPhysiol, 283:H1253-62[CrossRef]

Roubille F, Tardif JC. New therapeutic targets in cardiology: Heart failure and arrhythmia: HCN channels. Circulation 127(19), 1986-1996 (2013).

Schelbert, E. B. et al. Myocardial fibrosis quantified by extracellular volume is associated with subsequent hospitalization for heart failure, death, or both across the spectrum of ejection fraction and heart failure stage. J. Am. Heart Assoc. 4, e002613 (2015)

Schieffer B, Wirger A, Meybrunn M et al. Comparative effects of chronic angiotensin-converting enzyme inhibition and angiotensin II type 1 receptor blockade on cardiac remodeling after myocardial infarction in 
the rat. Circulation 89(5), 2273-2282 (1994).[CrossRef]

Sharma UC, Pokharel S, van Brakel TJ et al. Galectin-3 marks activated macrophages in failure-prone hypertrophied hearts and contributes to cardiac dysfunction. Circulation 110(19), 3121-3128 (2004).[CrossRef]

Shi Y, Massague J (2003). Mechanisms of TGF-beta signaling from cell membrane to the nucleus. Cell, 113:685$700[$ CrossRef]

Shinde AV, Frangogiannis NG. Fibroblasts in myocardial infarction: a role in inflammation and repair. J Mol Cell Cardiol. 2014;70:74-82

Shi-Wen X, Rodríguez-Pascual F, Lamas S, Holmes A, Howat S, Pearson JD, Dashwood MR, du Bois RM, Denton CP, Black CM, Abraham DJ, Leask A. Constitutive ALK5-independent c-Jun N-terminal kinase activation contributes to endothelin-1 overexpression in pulmonary fibrosis: evidence of an autocrineendothelin loop operating through the endothelin A and B receptors. Mol Cell Biol. 2006; 26:5518-5527. doi: 10.1128/ MCB.00625-06. 193

Simon, K., and A. Szepvolgyi. 1996. N-acetylcysteine in combination with nitroglycerin and streptokinase for the treatment of evolving acute myocardial infarction: safety and biochemical effects. Circulation 94:1791[CrossRef]

Skyschally, A., P. Gres, S. Hoffmann, M. Haude, R. Erbel, R. Schulz, et al. 2007. Bidirectional role of tumor necrosis factor-alpha in coronary microembolization: progressive contractile dysfunction versus delayed protection against infarction. Circ. Res. 100:140-146

S-L Puhl, A Kazakov, A Müller, P Fries, D RWagner, M Böhm, C Maack and Y Devaux , Adenosine A1 receptor activation attenuates cardiac hypertrophy and fibrosis in response to $\alpha 1$-adrenoceptor stimulation in vivo, British Journal of Pharmacology (2016) 173 88-102

Stein M, Boulaksil M, Jansen JA, Herold E, Noorman M, Joles JA, van Veen TA, Houtman MJ, Engelen MA, Hauer RN, de Bakker JM, van Rijen HV (2010). Reduction of fibrosis-related arrhythmias by chronic reninangiotensin-aldosterone system inhibitors in an aged mouse model. Am J Physiol Heart CircPhysiol, 299:H310-21

Sun M (2007) Tumor necrosis factor-alpha mediates cardiac remodeling and ventricular dysfunction after pressure overload state. Circulation 115: 1398-1407

Sun, Y., and Huang, P. (2016). Adenosine A2B receptor: from cell biology to human diseases. Front. Chem. 4:1329. doi: 10.4049/ jimmunol.0900515

Talasaz, A. H., H. Khalili, Y. Jenab, M. Salarifar, M. A. Broumand, and F. Darabi. 2013. N-Acetylcysteine effects on transforming growth factor-beta and tumor necrosis factor-alpha serum levels as pro-fibrotic and inflammatory biomarkers in patients following ST segment elevation myocardial infarction. Drugs R. D. 13:199-205

Thum T, Lorenzen JM. Cardiac fibrosis revisited by microRNA therapeutics. Circulation 126(7), 800-802 (2012). Bauersachs J. Regulation of myocardial fibrosis by micrornas. J. Cardiovasc. Pharmacol. 56(5), 454-459 (2010)

Travers JG, Kamal FA, Robbins J, Yutzey KE, Blaxall BC (2016) Cardiac Fibrosis: The Fibroblast Awakens. Circ Res 118: 1021-1040.

Tsutsui, H., S. Kinugawa, and S. Matsushima. 2011. Oxidative stress and heart failure. Am. J. Physiol. Heart Circ. Physiol. 301:H2181-H2190.

Turner NA, Porter KE, Smith WH, White HL, Ball SG, Balmforth AJ. Chronic beta2-adrenergic receptor stimulation increases proliferation of human cardiac fibroblasts via an autocrine mechanism. Cardiovasc Res. 2003;57:784-92[CrossRef]

Van Den Borne SW, Diez J, Blankesteijn WM, Verjans J (2010) Myocardial remodeling after infarction: the role of myofibroblasts. Nat Rev Cardiol 7: 30-37

vanRooij E, Sutherland LB, Thatcher JE, DiMaio JM, Naseem RH, Marshall WS, Hill JA, Olson EN. Dysregulation of microRNAs after myocardial infarction reveals a role of miR-29 in cardiac fibrosis. ProcNatlAcadSci U S A. 2008;105:13027-13032

Vecchio EA, White PJ and May LT (2017) Targeting Adenosine Receptors for the Treatment of Cardiac Fibrosis. Front. Pharmacol. 8:243. doi: 10.3389/fphar.2017.00243

Ventura-Clapier R, Garnier A, Veksler V (2008). Transcriptional control of mitochondrial biogenesis: the central role of PGC-1alpha. Cardiovasc Res, 79:208-17[CrossRef]

Vu TD, Pal SN, Ti LK, Martinez EC, Rufaihah AJ, Ling LH, et al. An autologous platelet-rich plasma hydrogel compound restores left ventricular structure, function and ameliorates adverse remodeling in a minimally invasive large animal myocardial restoration model: a translational approach: Vu and Pal "Myocardial Repair: PRP, Hydrogel and Supplements". Biomaterials. 2015; 45:27-35

Wakeno M, Minamino T, Seguchi O, Okazaki H, Tsukamoto O, Okada K, Hirata A, Fujita M, Asanuma H, Kim J, Komamura K, Takashima S, Mochizuki N, Kitakaze M: Long-term stimulation of adenosine A2b receptors 
begun after myocardial infarction prevents cardiac remodeling in rats. Circulation 2006, 114:1923-32

Wang, J.; Huang, W.; Xu, R.; Nie, Y.; Cao, X.; Meng, J.; Xu, X.; Hu, S.; Zheng, Z. MicroRNA-24 regulates cardiac fibrosis after myocardial infarction. J. Cell. Mol. Med. 2012, 16, 2150-2160.

Weber KT, Brilla CG (1992) Factors associated with reactive and reparative fibrosis of the myocardium. Basic Res Cardiol 87: 291-301

Worou, M. E., K. Belmokhtar, P. Bonnet, P. Vourc'h, M. C. Machet, G. Khamis, et al. 2011. Hemin decreases cardiac oxidative stress and fibrosis in a rat model of systemic hypertension via PI3K/Aktsignalling. Cardiovasc. Res. 91:320-329

Wynn TA. Common and unique mechanisms regulate fibrosis in various fibroproliferative diseases. J Clin Invest 2007; 117: 524-529

Xu X, Fassett J, Hu X, Zhu G, Lu Z, Li Y et al. (2008). Ecto-5'- nucleotidase deficiency exacerbates pressureoverload-induced left ventricular hypertrophy and dysfunction. Hypertension 51: 1557-1564

Yamamoto, S., G. Yang, D. Zablocki, J. Liu, C. Hong, S. J. Kim, et al. 2003. Activation of Mst1 causes dilated cardiomyopathy by stimulating apoptosis without compensatory ventricular myocyte hypertrophy. J. Clin. Invest. 111:1463-1474[CrossRef]

Yamauchi Y, Kohyama T, Takizawa H, Kamitani S, Desaki M, Takami K, et al. Tumor necrosis factor-alpha enhances both epithelial-mesenchymal transition and cell contraction induced in A549 human alveolar epithelial cells by transforming growth factor-beta1. Exp Lung Res. 2010;36:12-24[CrossRef][Pubmed]

Yang Y, Ago T, Zhai P, Abdellatif M, Sadoshima J (2011) Thioredoxin 1 negatively regulates angiotensin IIinduced cardiac hypertrophy through upregulation of miR-98/let-7. Circ Res 108: 305-313

Zeisberg EM, Tarnavski O, Zeisberg M, Dorfman AL, McMullen JR, Gustafsson E, Chandraker A, Yuan X, Pu WT, Roberts AB, Neilson EG, Sayegh MH, Izumo S and Kalluri R. Endothelial-to-mesenchymal transition contributes to cardiac fibrosis. Nat Med 2007; 13: 952-961[CrossRef][Pubmed]

Zhang Y, Huang XR, Wei LH, Chung AC, Yu CM, Lan HY. miR-29b as a therapeutic agent for angiotensin IIinduced cardiac fibrosis by targeting TGF-beta/Smad3 signaling. MolTher2014;22:974985[CrossRef][Pubmed]

Zhao, X.; Wang, K.; Liao, Y.; Zeng, Q.; Li, Y.; Hu, F.; Liu, Y.; Meng, K.; Qian, C.; Zhang, Q.; et al. MicroRNA101a inhibits cardiac fibrosis induced by hypoxia via targeting TGF_RI on cardiac fibroblasts. Cell. Physiol. Biochem. 2015, 35, 213-226. [CrossRef] [PubMed]

Zhaobo Fan and Jianjun Guan. Antifibrotic therapies to control cardiac fibrosis, Fan and Guan Biomaterials Research (2016) 20:13

Zhong X, Chung AC, Chen HY, Meng XM, Lan HY (2011) Smad3-mediated upregulation of miR-21 promotes renal fibrosis. J AmSocNephrol 22: 1668- 1681

Zhou L, Wang L, Lu L, Jiang P, Sun H, Wang H. Inhibition of miR-29 by TGF-beta-Smad3 signaling through dual mechanisms promotes transdifferentiation of mouse myoblasts into myofibroblasts. PLoS One 2012;7: e33766. doi: 10.1371/journal.pone.0033766 